\title{
5 Ergebnisse der explorativen Studie
}

Zwei zentrale Hypothesen werden im Literaturteil dieser Arbeit aufgestellt, nämlich die Hypothese der Formvariabilität (Kapitel 2: Welche phonetische Varianten treten auf?) und die Form-Kontext-Hypothese (Kapitel 3: Kann ein Teil der vorkommenden Formvariabilität durch den jeweiligen Kontext erklärt werden?). Im Folgenden werde ich mich diesen Fragen explorativ annähern.

\subsection{Füllpartikeln}

\subsubsection{Allgemeine und sprecherspezifische Häufigkeit}

Tabelle 5.1 gibt einen Überblick über die Anzahl der Füllpartikeln und ihre sprecherspezifische Auftretenshäufigkeit in den Dialogen aus GErman COnversations-Füllpartikeln v. 1 (GECO-FP). ${ }^{110}$ Die Sprechgeschwindigkeiten der Versuchspersonen rangieren zwischen 4,9 und 6,6 $\sigma / s$, die Anzahl der Füllpartikeln je Minute zwischen 1,4 und $4 \mathrm{FP} / \mathrm{min}$, mit einem Mittelwert von 2,9 FP/min. Es besteht weder eine signifikante Korrelation (nach Pearson) zwischen Sprechgeschwindigkeit und Füllpartikeln je Minute $\left(r=0,21, t=0,52, d f=6, p=0,6, \mathrm{KI}_{95 \%}=[-0,58 ; 0,8]\right.$ noch zwischen Tokenanzahl und Füllpartikelanzahl $(r=0,68, t=2,28, d f=6, p=0,1$, $\mathrm{KI}_{95 \%}=[-0,04 ; 0,94]$ und auch nicht zwischen Silbenanzahl und Füllpartikelanzahl $\left(r=0,67, t=2,22, d f=6, p=0,1, \mathrm{KI}_{95 \%}=[-0,06 ; 0,93]\right)$. Bei der geringen Sprecherinnenanzahl kann nicht mit Sicherheit angegeben werden, welcher Verteilung

\footnotetext{
${ }^{110}$ Die Tokenanzahl wurde zur Berechnung der Sprechgeschwindigkeit um 29 leere Token sowie um 1463 extralinguistische Phänomene wie Husten, Lachen, etc., um 12480 stille Pausen sowie um 111 Clicks reduziert. Enthalten sind jedoch Füllpartikeln, insbesondere auch glottale Füllpartikeln (annotiert mit $f g$ ), die orthographisch nicht wiederzugeben waren und auf der Ebene wordscor nur impressionistisch und ad hoc beschrieben werden. Ihre Unterscheidung in dieser Ebene ist für diese Studie nicht relevant. Dies sind die 101 Werte 〈brummen $\rangle(2),\langle\mathrm{G}\rangle(15),\langle$ knack $\rangle(11),\langle$ knacken $\rangle$

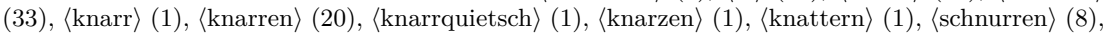
$\langle$ stimmlippen $\rangle(1),\langle$ stimmton $\rangle(6)$ und $\langle$ Stimmton $\rangle(1)$.
}

\section{Zusatzmaterial online}

Zusätzliche Informationen sind in der Online-Version dieses Kapitel (https://doi.org/10.1007/ 978-3-662-62812-6_5) enthalten. 
Tabelle 5.1: Artikulationsdauer je Versuchsperson (VP), Anzahl der Token, Silben $(\sigma)$ und Füllpartikeln (FP) sowie Sprechgeschwindigkeit $(\sigma / \mathrm{s})$, Prozent FP je Token und Silbe sowie FP je Minute für alle Versuchspersonen (VP) in GECO-FP. Zusätzlich sind die addierten Gesamtsummen $(\Sigma)$ sowie die Mittelwerte $(\bar{x})$ und Standardabweichung $(s)$ über alle VP angegeben.

\begin{tabular}{lrrrr|rccc}
\hline $\mathrm{VP}$ & $\Sigma \mathrm{min}$ & Token $^{\mathrm{a}}$ & Silben & $\sigma / \mathrm{s}$ & $\mathrm{FP}^{\mathrm{b}}$ & $\mathrm{FP} /$ Token $(\%)$ & $\mathrm{FP} / \sigma(\%)$ & $\mathrm{FP} / \mathrm{min}$ \\
\hline $\mathrm{A}$ & 19,03 & 4204 & 5821 & 5,10 & 30 & 0,71 & 0,52 & 1,58 \\
$\mathrm{C}$ & 23,69 & 4731 & 6904 & 4,86 & 80 & 1,69 & 1,16 & 3,38 \\
$\mathrm{D}$ & 21,95 & 4974 & 6796 & 5,16 & 86 & 1,73 & 1,27 & 3,92 \\
$\mathrm{~F}$ & 18,69 & 4058 & 5678 & 5,06 & 27 & 0,67 & 0,48 & 1,44 \\
$\mathrm{H}$ & 19,63 & 4956 & 7104 & 6,03 & 79 & 1,59 & 1,11 & 4,02 \\
$\mathrm{~J}$ & 26,45 & 7197 & 10427 & 6,57 & 61 & 0,85 & 0,59 & 2,31 \\
$\mathrm{~K}$ & 32,10 & 8189 & 11600 & 6,02 & 120 & 1,47 & 1,03 & 3,74 \\
$\mathrm{M}$ & 25,69 & 5900 & 8834 & 5,73 & 75 & 1,27 & 0,85 & 2,92 \\
\hline$\Sigma$ & 187,2 & 44209 & 63164 & - & 558 & - & - & - \\
$\bar{x}$ & 23,4 & 5526,1 & 7895,5 & 5,6 & 69,8 & 1,2 & 0,9 & 2,9 \\
$s$ & 4,6 & 1473 & 2172,9 & 0,6 & 30,5 & 0,4 & 0,3 & 1 \\
\hline
\end{tabular}

${ }^{\text {a }}$ Ohne leere Token, extralinguistische Phänomene, stille Pausen und Clicks.

${ }^{\mathrm{b}}$ Alle mit $f v$ und $f g$ annotierte Füllpartikeln.

die Daten folgen. Tests auf Normalverteilung werden erst ab ca. 50 Datenpunkten robust. Somit kann hier nicht ausgeschlossen werden, dass die zugrundeliegende Verteilung der Datenpunkte von der Normalverteilung abweicht. ${ }^{111}$ Tabelle 5.2 enthält die glottalen und nicht-glottalen Füllpartikelformen je Versuchsperson.

Tabelle 5.2: Anzahl der glottalen $(f g)$ und nicht-glottalen $(f v)$ Füllpartikeln je Versuchsperson in GECO-FP.

\begin{tabular}{r|rrrrrrrrr|r}
\hline & $\mathrm{A}$ & $\mathrm{C}$ & $\mathrm{D}$ & $\mathrm{F}$ & $\mathrm{H}$ & $\mathrm{J}$ & $\mathrm{K}$ & $\mathrm{M}$ & $\Sigma$ & $\%$ \\
\hline $\mathrm{fg}$ & 13 & 24 & 17 & 3 & 6 & 1 & 38 & 9 & 111 & 19,89 \\
$\mathrm{fv}$ & 17 & 56 & 69 & 24 & 73 & 60 & 82 & 66 & 447 & 80,11 \\
\hline$\Sigma$ & 30 & 80 & 86 & 27 & 79 & 61 & 120 & 75 & 558 & \\
\hline
\end{tabular}

Auch hier kann aufgrund der Stichprobengröße nicht mit Sicherheit angegeben werden, welcher Verteilung die Daten folgen. ${ }^{112}$ Fast $20 \%$ aller Füllpartikelformen

\footnotetext{
${ }^{111}$ Ein Shapiro-Wilk-Test auf Normalverteilung ergibt keine Anzeichen, dass die Anzahl der Füllpartikeln über alle Versuchspersonen nicht normalverteilt ist $(W=0,93, p=0,5)$. Selbiges gilt für die Anzahl an Füllpartikeln je Minute $(W=0,89, p=0,2)$ und den prozentualen Anteil in Füllpartikeln $(W=0,87, p=0,1)$.

${ }^{112}$ Über alle Versuchspersonen zeigen auch diese Verteilungen weder für die glottalen Füllpartikeln ( $W=0,91, p=0,4)$ noch für die nicht-glottalen Füllpartikeln $(W=0,87, p=0,1)$ Abweichungen
} 
in GECO-FP sind glottale Füllpartikeln.

\subsubsection{Segmentale Formen}

\subsubsection{Nicht-glottale Formen}

Auf der Ebene segm sind 67 verschiedene Formen von Segmentabfolgen vorhanden. Durch Zusammenfassung konzeptuell ähnlicher Segmentabfolgen kann die Anzahl der Formen auf 55 segmental-abstrahierte Formen reduziert werden. Tabelle 5.3 zeigt die zehn häufigsten segmental-abstrahierten Formen (für alle segmentalabstrahierten Formen s. Tabelle D.2 im Anhang). Am häufigsten kommen ?VN, ?V, $\mathrm{VN}$ und $\mathrm{V}$ vor, also vokalische und vokalisch-nasale Formen mit und ohne vorangehendem glottalen Plosiv. Weniger häufig sind Formen, in denen dem Vokal eine Sequenz glottaler Plosive vorausgeht (GVN, GV).

Tabelle 5.3: Anzahl der zehn häufigsten segmental-abstrahierten Formen aller als $f v$ annotierten Füllpartikeln in GECO-FP (? = ein bis drei glottale Plosive, G = Sequenz von mehr als drei glottalen Plosiven, $\mathrm{V}=$ Vokal, $\mathrm{N}=$ Nasal).

\begin{tabular}{lr}
\hline Segmente & Anzahl \\
\hline ?VN & 105 \\
?V & 76 \\
VN & 51 \\
V & 50 \\
GVN & 33 \\
GV & 23 \\
N & 15 \\
?VNG & 7 \\
?VC & 5 \\
?VN? & 5 \\
\hline
\end{tabular}

Abbildung 5.1 zeigt die Verteilung der abstrahierten Segmentformen und ihre Darstellung auf einer Log-log-Skala. Die logarithmisierten Häufigkeitswerte und ihre Ränge nähern sich einer Geraden mit negativer Steigung $(y=-x)$ an, was auf eine Zipfverteilung der Daten deutet (Zipf 1949). Segmentale Füllpartikelformen verhalten sich somit ähnlich wie viele andere sprachliche Phänomene.

Die orthographische Repräsentation ist eine weitere Abstraktion der phonetisch vorhandenen Segmente. Abbildung 5.2 zeigt die Relation der segmental-abstrahierten von der Normalverteilung (Shapiro-Wilk-Test auf Normalverteilung). 


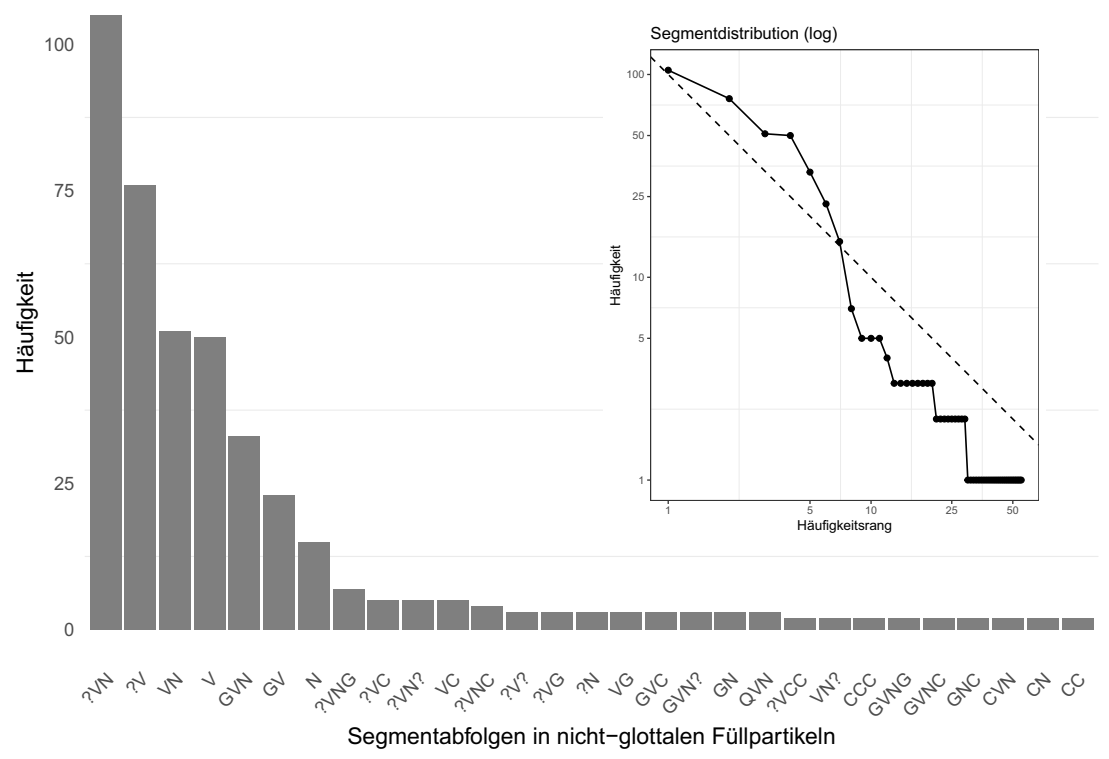

Abbildung 5.1: Anzahl $(n>1)$ der abstrahierten Segmentabfolgen in Füllpartikeln $(?=$ ein bis drei glottale Plosive, $\mathrm{G}=$ Sequenz von mehr als drei glottalen Plosiven, $\mathrm{V}=$ Vokal, $\mathrm{N}=$ Nasal, $\mathrm{C}=$ weitere Konsonanten und Clicks, $\mathrm{Q}=$ hohe gepresste Stimme). Der innere Graph enthält alle Rangfrequenzen der Füllpartikelformen (logarithmisch) und eine ideale Zipf-Verteilung (gestrichelt).

Formen zu den orthographischen Repräsentationen. ${ }^{113}$ In Abbildung 5.2 lässt sich gut erkennen, dass die als $\ddot{a} h$, ähm und $h m$ wiedergegebenen orthographischen Formen phonetisch divers sind. Besonders zu Anfang und Ende einer Füllpartikel treten ein oder mehrere glottale Plosive auf. Die häufigsten $\mathrm{hm}$-Formen in dieser Datenstichprobe enthalten keinen glottalen Frikativ [h]. Die drei häufigsten orthographischen Vorkommen sind ähm (224 mal), äh (179 mal) und hm (20 mal). ähm kommt somit 1,3 mal häufiger vor als äh, was signifikant häufiger ist (zweiseitiger exakter Binomialtest, $\mathrm{p}<0,05)$. Dies deckt sich mit früheren Studien, in denen Frauen

\footnotetext{
${ }^{113}$ Für alle Formen, die mehr als fünf Vorkommen haben (was 98 abstrahierte Formen beziehungsweise $21,9 \%$ der 447 nicht-glottalen Füllpartikeln ausschließt). Orthographisch ist $m$ und $m h$ zur , gängigen' Form hm zusammengefasst worden.
} 


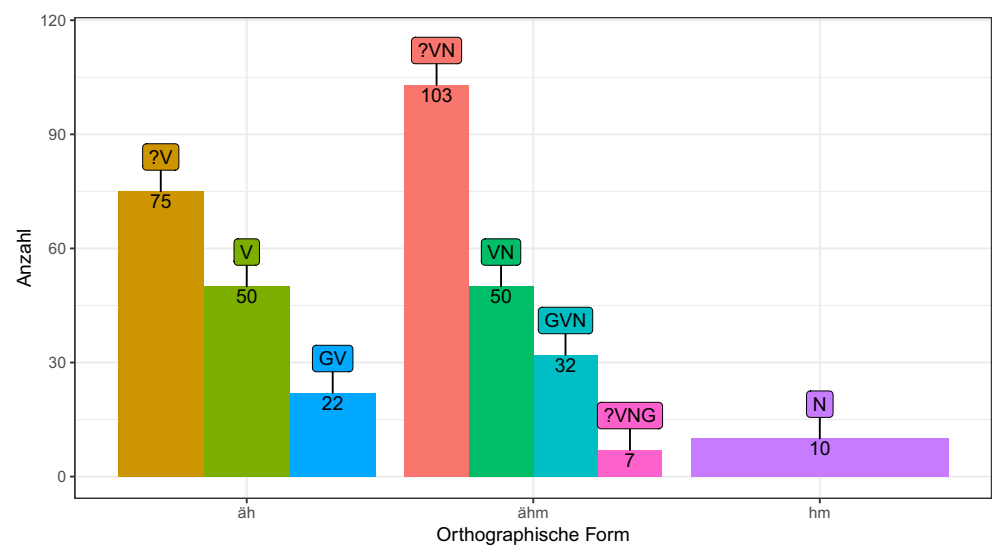

Abbildung 5.2: Zusammenfassung verschiedener segmentaler Realisierungen $(n>5)$ unter zwei orthographischen Formen (? = ein bis drei glottale Plosive, $\mathrm{G}=$ Sequenz von mehr als drei glottalen Plosiven, $\mathrm{V}=$ Vokal, $\mathrm{N}=$ Nasal, $\mathrm{C}=$ Konsonant).

mehr VN-Formen als V-Formen produzieren (vgl. Wieling et al. 2016). Da alle Versuchspersonen in GECO-FP weiblich sind, kann dieses Verhältnis allerdings erst in der nächsten Studie mit Männern verglichen werden. Offen bleibt die Frage, wieso Frauen häufiger VN-Formen als V-Formen verwenden.

\subsubsection{Glottale Formen}

Für die glottalen Formen abstrahiere ich Segmentabfolgen mit mehr als einem glottalen Plosiv und perzeptiv deutlich abgesetzten (im akustischen Signal mit Stille $>50 \mathrm{~ms}$ markierten) Sequenzen zu ?+ (beispielsweise[21?]). Laut Tabelle D.3 im Anhang bestehen die häufigsten glottalen Füllpartikelformen aus einer engen Abfolge glottaler Plosive (G), aus singulären glottalen Plosive (?) und aus einer losen, perzeptiv vereinzelt wahrnehmbaren Abfolge glottaler Plosive $(?+)$. Wie schon bei den nicht-glottalen Formen zeigt auch die visuelle Inspektion der segmentalabstrahierten glottalen Füllpartikelformen eine Distribution, die eine Zipf-Verteilung vermuten lässt (vgl. Abbildung 5.3). 


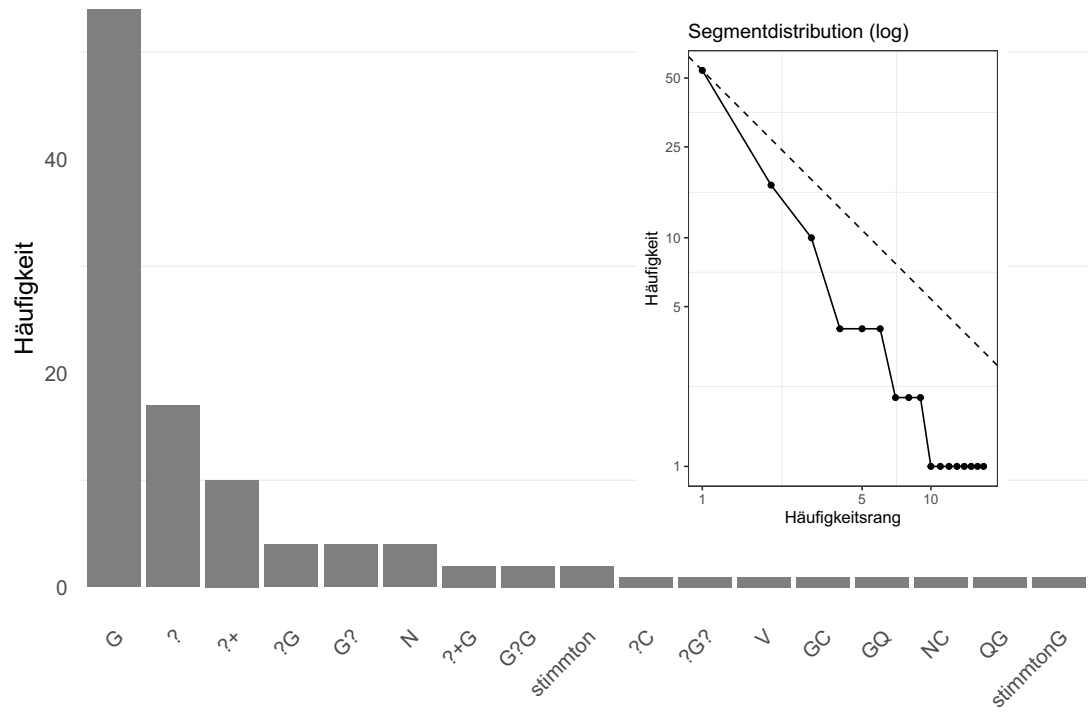

Segmentabfolgen in glottalen Füllpartikeln

Abbildung 5.3: Anzahl der abstrahierten glottalen Segmentabfolgen in Füllpartikeln (? $=$ ein bis drei glottale Plosive, $?+=$ perzeptiv vereinzelt wahrnehmbare Abfolge glottaler Plosive, $\mathrm{G}=$ Sequenz von mehr als drei glottalen Plosiven, $\mathrm{V}=$ Vokal, $\mathrm{N}=$ Nasal, $\mathrm{C}=$ Konsonant, $\mathrm{Q}=$ hohe gepresste Stimme, stimmton = Stimmton). Der innere Graph enthält alle Rangfrequenzen der Füllpartikelformen (logarithmisch) und eine ideale Zipf-Verteilung (gestrichelt).

\subsubsection{Dauer}

\subsubsection{Nicht-glottale Formen}

Die geringste Dauer einer nicht-glottalen Füllpartikel in GECO-FP beträgt 34,6 ms $(? \rightarrow$ E beziehungsweise abstrahiert ?V $)$, die längste $1239,6 \mathrm{~ms}(? \rightarrow \mathrm{E} \rightarrow \mathrm{p} \rightarrow \mathrm{f}$ beziehungsweise abstrahiert ?VCC). Abbildung 5.4 zeigt die Distribution der auf Silben normalisierten nicht-glottalen Füllpartikeln für die häufigsten Formen $(n>5)$. Zur Berechnung der normalisierten Dauer vgl. Abschnitt 4.4.1.

Aus Abbildung 5.4 lässt sich eine positive Korrelation (nach Pearson) zwischen Segmentanteilen in der phonetischen Realisierung und der Länge von Füllpartikeln 


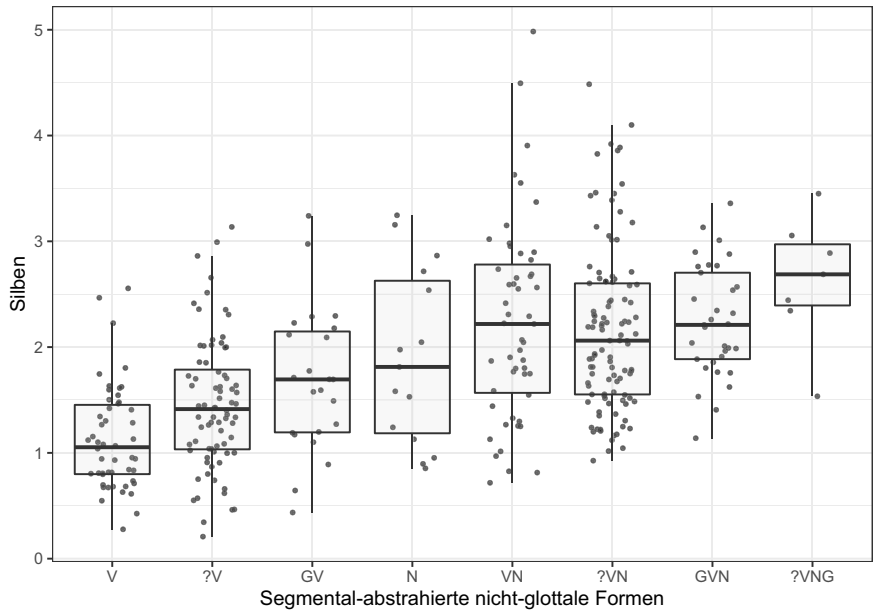

Abbildung 5.4: Dauer (in Silben) der häufigsten $(n>5)$ segmental-abstrahierten nichtglottalen Füllpartikelformen in GECO-FP (? = ein bis drei glottale Plosive, G = Sequenz von mehr als drei glottalen Plosiven, $\mathrm{V}=$ Vokal, $\mathrm{N}=$ Nasal).

ableiten $\left(r=0,42, t=9,83, d f=445, p<0,001, \mathrm{KI}_{95 \%}=[0,34 ; 0,5]\right)$. Je mehr Segmente phonetisch realisiert werden, desto länger ist ihre Dauer. Mithife von bonferronikorrigierten Post-hoc-Tests werden die Formen ermittelt, die sich signifikant voneinander unterscheiden (vgl. Tabelle 5.4).

Tabelle 5.4: Bonferronikorrigierte p-Werte für paarweise t-Tests der Mittelwerte der acht segmental-abstrahierten nicht-glottalen Füllpartikelformen in Abbildung 5.4.

\begin{tabular}{l|rrrrrrr}
\hline & $\mathrm{V}$ & ?V & GV & N & VN & ?VN & GVN \\
\hline ?V & 0,353 & - & - & - & - & - & - \\
GV & 0,057 & 1 & - & - & - & - & - \\
$\mathrm{N}$ & $<0,01$ & 0,757 & 1 & - & - & - & - \\
VN & $<0,001$ & $<0,001$ & 0,063 & 1 & - & - & - \\
?VN & $<0,001$ & $<0,001$ & 0,129 & 1 & 1 & - & - \\
GVN & $<0,001$ & $<0,001$ & 0,12 & 1 & 1 & 1 & - \\
?VNG & $<0,001$ & $<0,01$ & 0,066 & 0,735 & 1 & 1 & 1 \\
\hline
\end{tabular}

Die V-Form ist signifikant kürzer als die vokalisch-nasalen Formen VN, ?VN, GVN,?VNG und die nasale Form N. Die Form mit vorangehendem glottalen Plosiv 
?V ist immer noch signifikant kürzer als alle vokalisch-nasalen Formen. Die GVForm (mit vorangehenden Sequenzen glottaler Plosive) ist in ihrer Dauer nicht von den anderen Formen zu unterscheiden. Während also prinzipiell denkbar wäre, dass eine V-Form mit gleicher Dauer wie eine VN-Form geäußert wird, zeigen die Daten doch einen systematische Unterscheidung beider Formen bezüglich ihrer Dauer. Der Vollständigkeit halber und zum Vergleich mit anderen Studien gibt Tabelle 5.5 die durchschnittliche Dauer der acht häufigsten Formen in der auf Sprechgeschwindigkeit normalisierten Dauer in Silben und der nicht normalisierten Dauer in Millisekunden an. Insgesamt haben V-Formen haben eine durchschnittliche Dauer von $\bar{x}=1,5 \sigma$ $(s=0,7 \sigma)$, VN-Formen von $\bar{x}=2,3 \sigma(s=0,8 \sigma)$ und N-Formen von $\bar{x}=2,5 \sigma$ $(s=1,1 \sigma)$.

Tabelle 5.5: Mittelwerte und Standardabweichungen der normalisierten Dauer in Silben $(\sigma)$ pro Sekunde und der nicht-normalisierten Dauer (in Millisekunden) der acht häufigsten segmental-abstrahierten nicht-glottalen Füllpartikelformen in Abbildung 5.4.

\begin{tabular}{l|rr|rr}
\hline Form & $\bar{x}($ in $\sigma)$ & $\mathrm{s}($ in $\sigma)$ & $\bar{x}$ (in ms) & $\mathrm{s} \mathrm{(in} \mathrm{ms)}$ \\
\hline V & 1,1 & 0,5 & 205,6 & 93,0 \\
?V & 1,5 & 0,6 & 256,7 & 110,9 \\
GV & 1,7 & 0,7 & 291,4 & 108,1 \\
N & 1,9 & 0,8 & 334,4 & 150,4 \\
?VN & 2,2 & 0,8 & 371,5 & 149,1 \\
VN & 2,2 & 0,9 & 381,9 & 127,2 \\
GVN & 2,2 & 0,5 & 393,4 & 94,1 \\
?VNG & 2,6 & 0,6 & 473,5 & 124,0 \\
\hline
\end{tabular}

Die nicht-normalisierten Mittelwerte und Standardabweichungen für die orthographischen Formen ( $\ddot{a} h: \bar{x}=262,2, s=121 ; \ddot{a} h m: \bar{x}=395,8, s=139,5$; hm: $\bar{x}=450,4, s=182,6$; in Millisekunden) in GECO-FP können dank Angabe von Stichprobengröße, Mittelwerten und Standardabweichungen mit denen von Leeuw (2007) verglichen werden (vgl. Tabelle 2.5). Keine der drei Formen weicht signifikant voneinander ab (t-Test mit ungleichen Stichprobengrößen und ungleichen Varianzen nach Welch-Satterthwaite), womit die durchschnittliche unnormalisierte Dauer von Füllpartikeln in den Interviews von Leeuw repliziert wird.

In der Literatur wird ein möglicher Effekt der Vokaldauer in Abhängigkeit von der Füllpartikelform angesprochen (Jessen 2012). Zur Überprüfung rechne ich ein lineares gemischtes Modell mit Dauer als abhängiger Variable und Form als unabhängiger Variable. Versuchspersonen gehen als Random Intercepts ein, mit Random 
Slopes für die Form je Versuchsperson. Abbildung 5.5 visualisiert die Vorhersagen des Modells, Tabelle 5.6 enthält die Werte. Das Modell wird folgendermaßen in R dargestellt.

1 lmer (Vokaldauer $\sim$ Form $+(1+$ Form $\mid \mathrm{VP})$, data ...

Die Vokaldauer in vokalisch-nasalen Füllpartikeln ist mit -68,6 ms tatsächlich signifikant kürzer als in vokalischen Füllpartikeln.

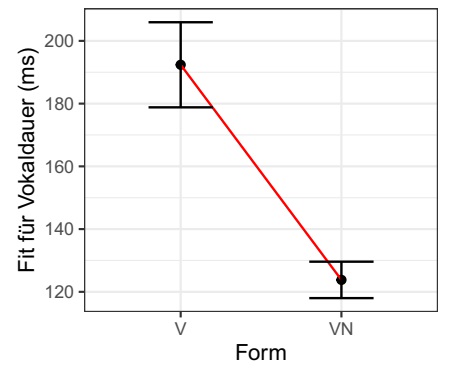

Abbildung 5.5: Effekt für die Vokaldauer in Abhängigkeit von der Füllpartikelform in GECO-FP.

Tabelle 5.6: Linear gemischtes Modell für die Vokaldauer in Abhängigkeit von Form + $\left(1+\right.$ Form/VP), mit erklärter Varianz $R^{2}$.

\begin{tabular}{lc}
\hline & Vokaldauer \\
\hline (Intercept) & $192,4(13,6)^{* * *}$ \\
FormVN & $-68,6(12,8)^{* * *}$ \\
\hline AIC & 4381,0 \\
Num. obs. & 391 \\
Num. groups: vp & 8 \\
\hline $\mathrm{R}_{m}^{2} / \mathrm{R}_{c}^{2}$ & $0,2 / 0,29$ \\
\hline${ }^{* * *} p<0,001,{ }^{* *} p<0,01,{ }^{*} p<0,05$
\end{tabular}

\subsubsection{Glottale Formen}

Die häufigsten glottalen Formen mit mehr als fünf Vorkommen sind ?, G und ?+. Die Form ? ist signifikant kürzer als die Form G und ?+ (paarweiser bonferronikorrigierter t-Test, jeweils $p<0,001)$. Abbildung 5.6 zeigt ihre Distribution, während Ta- 
belle 5.7 die auf Silben normalisierten sowie die nicht-normalisierten Werte auflistet. Der geringste Wert einer glottalen Füllpartikel liegt bei $27 \mathrm{~ms}(? \rightarrow \mathrm{G}$ beziehungsweise abstrahiert ?G), der größte Wert bei $1068,4 \mathrm{~ms}(\mathrm{G})$.

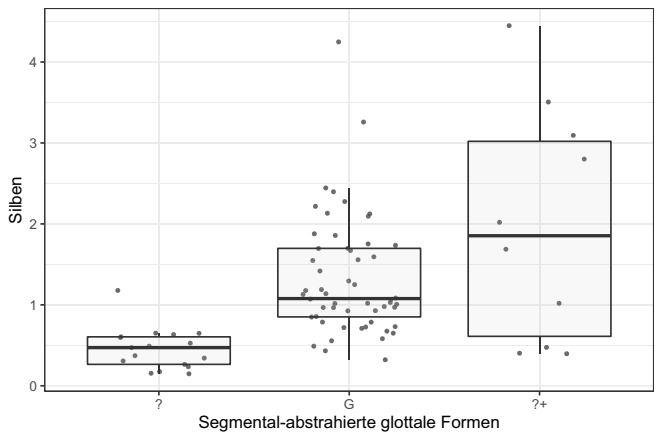

Abbildung 5.6: Dauer (in Silben) der häufigsten $(n>5)$ segmental-abstrahierten glottalen Füllpartikelformen in GECO-FP (? = ein bis drei glottale Plosive, G = Sequenz von mehr als drei glottalen Plosiven, $?+=$ Sequenz vereinzelt perzipierbarer glottaler Plosive mit intersegmentalen Pausen $>50 \mathrm{~ms}$ ).

Tabelle 5.7: Mittelwerte und Standardabweichungen der normalisierten Dauer (in Silben $\sigma)$ und der nicht-normalisierten Dauer (in Millisekunden) der drei häufigsten segmentalabstrahierten glottalen Füllpartikelformen in Abbildung 5.6.

\begin{tabular}{l|rr|rr}
\hline Form & $\bar{x}($ in $\sigma)$ & $\mathrm{s}($ in $\sigma)$ & $\bar{x}$ (in ms) & $\mathrm{s}($ in $\mathrm{ms})$ \\
\hline$?$ & 0,5 & 0,3 & 80,7 & 50,2 \\
$\mathrm{G}$ & 1,3 & 0,7 & 239,6 & 133,8 \\
$?+$ & 2,0 & 1,4 & 347,2 & 236,5 \\
\hline
\end{tabular}

\subsubsection{Grundfrequenz}

Für die Analyse der Grundfrequenz $\left(f_{0}\right)$ werden nur die nicht-glottalen Formen betrachtet, da in den glottalen Formen die Stimmlippenschwingung zu unregelmäßig für die reliable Ermittlung der Grundfrequenz ist. Die Grundfrequenz messe ich in der Mitte der Füllpartikel, wobei vorangehende und nachfolgende mögliche andere Lautsegmente nicht berücksichtigt werden. Dennoch kann es zu Fällen kommen, in denen die Grundfrequenz in Teilen nicht ermittelt werden kann. Zur ersten Übersicht 
zeigt das Histogramm in Abbildung 5.7 die Häufigkeit der Grundfrequenz über die Anzahl der vokalischen und vokalisch-nasalen Füllpartikeln. In 28 Fällen misst der Algorithmus $0 \mathrm{~Hz}$. Die restlichen Daten zerfallen in zwei Gruppen; eine erste zwischen 0 und $150 \mathrm{~Hz}$ (der Mittelwert dieser Häufung beträgt 95,2) und eine zweite ab $150 \mathrm{~Hz}$ (der Mittelwert beträgt 202,9). Möglicherweise sind die Daten in der ersten Gruppe auf glottalisierte Sequenzen zurückzuführen.

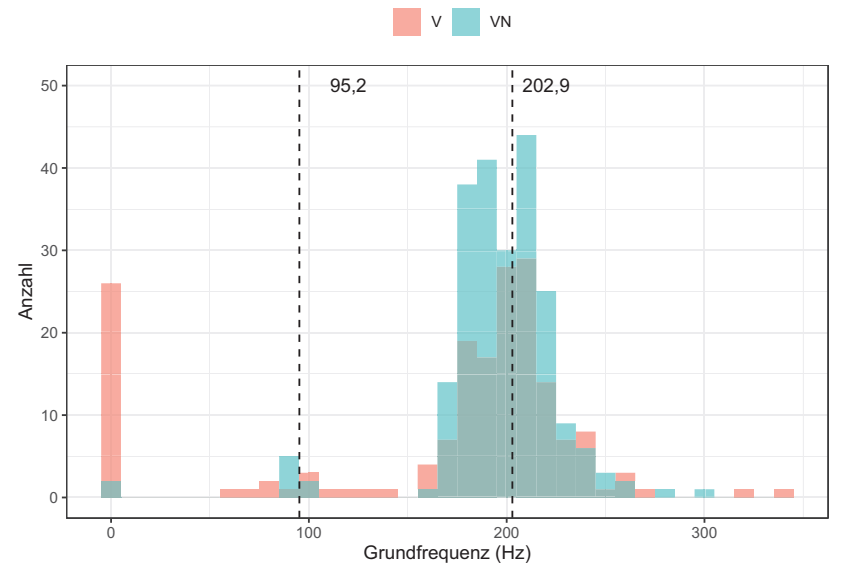

Abbildung 5.7: Histogramm der im Mittelpunkt aller V- (rot) und VN-Formen (blau) gemessenen Grundfrequenz mit einer Klassenbreite von $10 \mathrm{~Hz}$ und den Mittelwerten der beiden Häufungen (gestrichelte Linien) in GECO-FP.

Die Heuristik, in der Mitte eines Wortes beziehungsweise einer Füllpartikel zu messen, kann bei solchen glottalisierten oder laryngalisierten Sequenzen dazu führen, dass zufällig ein solcher Abschnitt in den Messpunkt fällt, der dann als Grundfrequenz der gesamten Füllpartikel interpretiert wird. Abbildung 5.8 veranschaulicht dies exemplarisch an allen Füllpartikelgrundfrequenzverläufen von Sprecherin C. Somit kann die aus dem Histogramm in Abbildung 5.7 inferierte Dichotomie zweier Häufungen nicht aufrechterhalten werden, da es sich bei den Fällen, welche bei Sprecherin C die erste Gruppe zwischen 0 und $150 \mathrm{~Hz}$ konstituieren, fast ausschließlich um eine zufälligerweise am Punkt der Messung vorhandene tiefe Grundfrequenz (Abbildung $5.7 i d=20$ ) oder um Interpolierungen (Abbildung $5.7 i d=51,58$ ) handelt und nicht um komplette Verläufe. Zwar könnte für jede Versuchsperson ein idealisierter 


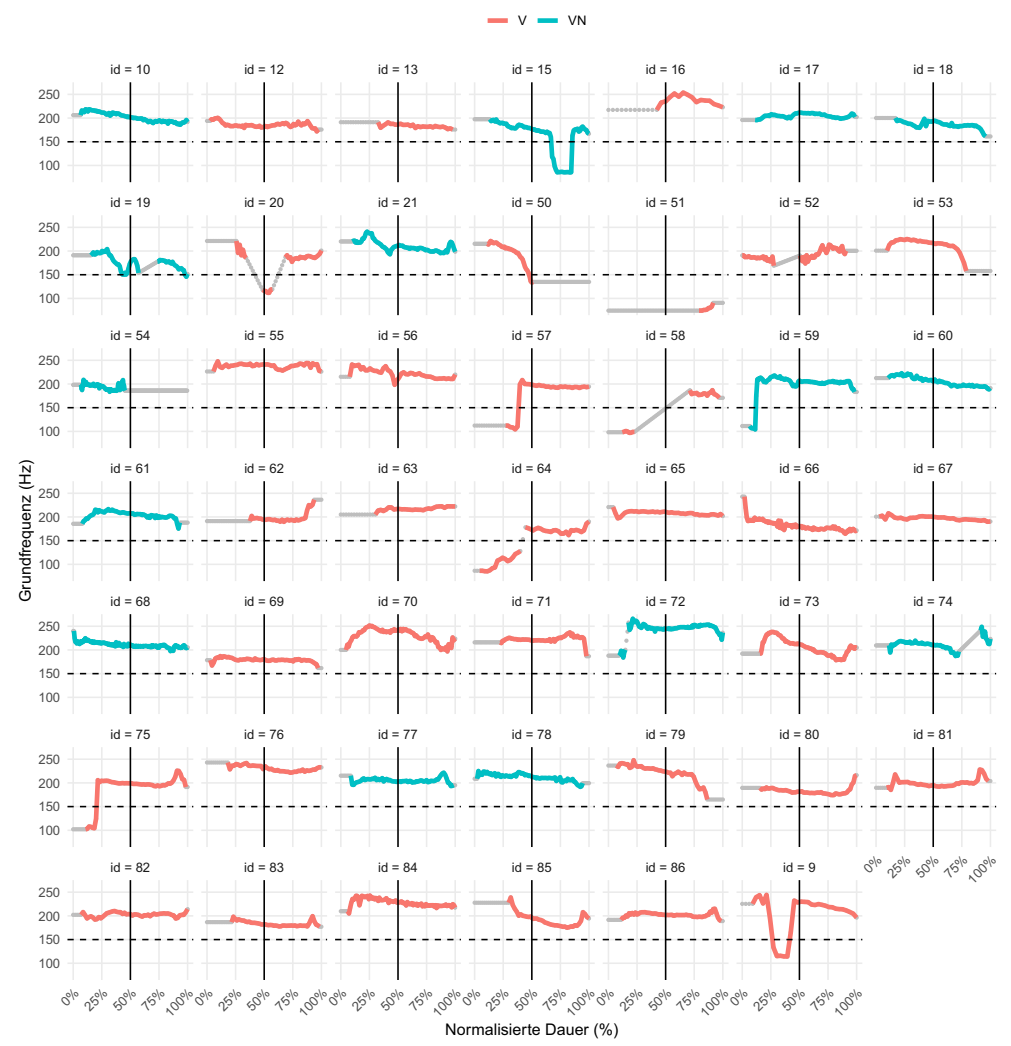

Abbildung 5.8: Grundfrequenzverläufe aller Segmente in V- und VN-Formen von Sprecherin $\mathrm{C}$ in GECO-FP mit normalisierter Dauer. Graue Punkte sind interpolierte Daten, die ursprünglich fehlen. Die vertikale Linie kennzeichnet den Messpunkt für die Grundfrequenz, die horiontale gestrichelte Linie markiert $150 \mathrm{~Hz}$.

Grundfrequenzverlauf mithilfe der Interpolierung fehlender Daten erzeugt werden, die Aussagekraft dieser Verläufe wäre jedoch nur schwer interpretierbar, da auch fallende, progrediente, steigende oder polynom verlaufende Konturen in eine Kurve gefittet würden. Daher nehme ich im Folgenden auch Abstand von der Analyse der Grundfrequenztrajektorien und beschränke mich auf im Mittelpunkt gemessenen Werte, jedoch ohne Interpolierung. 
Zur Einordnung der Grundfrequenzdaten wird die Grundfrequenz aller Füllpartikeln je Versuchsperson $\left(\bar{f}_{0 v p}^{F P}\right)$ mit der mittleren Grundfrequenz je Versuchsperson $\left(\bar{f}_{0 v p}\right)$ verglichen, i. e. die über alle Äußerungen einer Versuchsperson gemittelte Grundfrequenz (vgl. Braun \& Rosin 2015). Anders als in bisherigen Studien ist es nach der Sichtung des Histogramms in Abbildung 5.7 jedoch geboten, nur Grundfrequenzen über $150 \mathrm{~Hz}$ zu betrachten, um mögliche Messfehler oder möglicherweise starke Glottalisierungen auszuschließen. Ein genauer Wert zur Trennung zwischen solcher modaler und ,anderer' Phonation ist jedoch immer arbiträr und erfordert die Betrachtung aller mittleren Messpunkte aller Wörter aller Versuchspersonen, wie sie in Abbildung 5.9 dargestellt ist. Nach dieser Visualisierung kann die Schwelle aufgrund der eher niedrigeren Grundfrequenz von Sprecherin K auf $140 \mathrm{~Hz}$ abgesenkt werden. Tabelle 5.8 zeigt die Anzahl der Füllpartikeln und Wörter über und unter dieser Schwelle für alle Sprecherinnen. Die Schwelle von $140 \mathrm{~Hz}$ schließt 11,7\% aller V- und VN-Füllpartikeln aus.

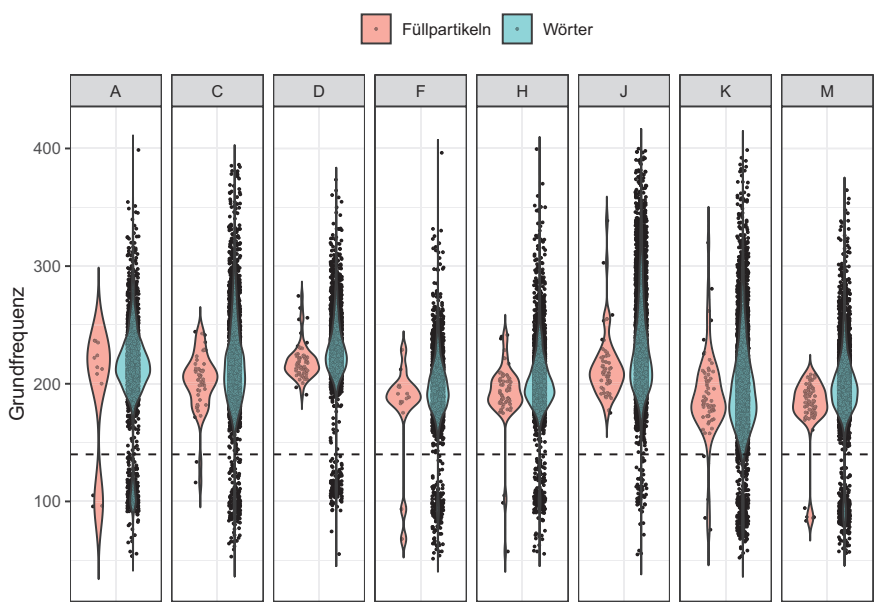

Abbildung 5.9: Mittlere Grundfrequenz für Füllpartikeln und Wörter je Versuchsperson in GECO-FP (im Mittelpunkt gemessen). Die horiontale gestrichelte Linie markiert $140 \mathrm{~Hz}$. Die Violinen zeigen die Häufigkeitsverteilung der Messpunkte (je breiter, desto mehr Messpunkte sind enthalten).

Tabelle 5.9 stellt die Mittelwerte und Standardabweichungen der Einzelmesswer- 
Tabelle 5.8: Anzahl der in der Mitte gemessenen V- und VN-Füllpartikeln und Wörter je Grundfrequenzschwelle in GECO-FP.

\begin{tabular}{l|rr}
\hline Grundfrequenz & Füllpartikeln & Wörter \\
\hline $0 \mathrm{~Hz}$ & 28 & 12078 \\
$>0$ und $<140 \mathrm{~Hz}$ & 355 & 30647 \\
$\geq 140 \mathrm{~Hz}$ & 19 & 1382 \\
\hline$\Sigma$ & 402 & 44107 \\
\hline
\end{tabular}

te der beiden Füllpartikelgrundfrequenzgruppen und die über alle Äußerungen einer Versuchsperson gemittelte $\bar{f}_{0 v p}$ mit dem gleichen Wertebereich dar. Eine Evaluierung erfolgt mithilfe paarweiser Vergleiche eines gerichteten t-Tests. ${ }^{114}$ Ein gerichteter Test wird durchgeführt, da die in der Literatur gefundenen Werte bisher häufig eine niedrigere $\bar{f}_{0 v p}^{F P}$ ergaben als die mittlere Grundfrequenz einer Versuchsperson (vgl. Braun \& Rosin 2015; Klug 2013). Füllpartikeln $(n=402)$ zeigen für den Bereich oberhalb von $140 \mathrm{~Hz}$ tatsächlich signifikant niedrigere Grundfrequenzen als die restlichen Äußerungen $(n=44107)$ nach Versuchsperson oberhalb von $140 \mathrm{~Hz}(t=-4,3$, $p<0,01, \mathrm{KI}_{95 \%}=[-$ Inf $\left.;-5,32]\right)$, bis auf Sprecherin A.

Tabelle 5.9: Mittelwerte und Standardabweichung für die in der Mitte von Füllpartikeln $\left(\bar{f}_{0 v p}^{F P}\right)$ und von allen Wörtern $\left(\bar{f}_{0 v p}\right)$ einer Versuchsperson gemessene Grundfrequenz in Hertz in GECO-FP.

\begin{tabular}{l|rr|rr}
\hline & \multicolumn{2}{|c|}{ Füllpartikeln } & \multicolumn{2}{|c}{ Wörter } \\
VP & $\bar{x}$ & $s$ & $\bar{x}$ & $s$ \\
\hline $\mathrm{A}$ & 221,1 & 13,3 & 220,1 & 26,2 \\
$\mathrm{C}$ & 205,3 & 18,8 & 220,2 & 37,3 \\
$\mathrm{D}$ & 218,9 & 15,0 & 230,7 & 23,7 \\
$\mathrm{~F}$ & 194,0 & 13,9 & 199,1 & 22,6 \\
$\mathrm{H}$ & 196,2 & 15,8 & 204,3 & 25,7 \\
$\mathrm{~J}$ & 215,1 & 28,4 & 232,0 & 42,9 \\
$\mathrm{~K}$ & 195,8 & 30,7 & 201,0 & 39,8 \\
$\mathrm{M}$ & 186,5 & 11,7 & 201,2 & 26,2 \\
\hline
\end{tabular}

\footnotetext{
${ }^{114}$ Die Wahl des t-Tests statt des nicht-parametrischen Wilcoxon-Rangsummentest wird durch die Normalverteilung der Differenzen der zu vergleichenden Werte motiviert. Somit kann der mächtigere t-Test verwendet werden.
} 


\subsubsection{Formanten}

Zur Ermittlung der Formanten und damit der Vokalqualität der Füllpartikeln extrahiere ich aus jeder Füllpartikel einer Versuchsperson $F_{1}$ und $F_{2}$ in der Mitte des Vokals; ebenso für die Referenzvokale. Alle Formanten wurden zuvor manuell in der EMU-Datenbank korrigiert. Alle Referenzvokale einer Qualität werden je Versuchsperson zu einem einzigen Vertreter dieser Klasse gemittelt. Anschließend wird mit den Werten der Referenzvokale und der Füllpartikelvokale eine formant-intrinsische, sprecher-intrinsische und vokal-extrinsische Lobanovnormalisierung durchgeführt (vgl. Abschnitt 4.4.3). Die so normalisierten Vokalwerte werden für $F_{1}$ und $F_{2}$ in Abbildung 5.10 in einem Vokalraum dargestellt, der die Streuung der im quasi-stationären Vokalteil (50\%) gemessenen Referenz und Füllpartikelvokale als Datenellipsen zeigt.

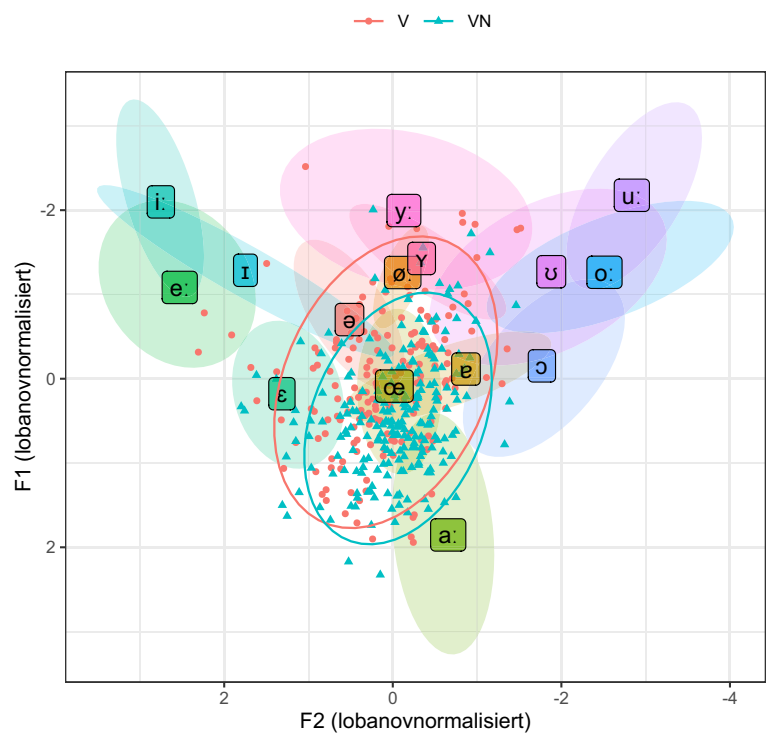

Abbildung 5.10: Mittig gemessene Vokale in V- und VN-Formen (Datenellipsen beschreiben $95 \%$ aller Messwerte) im lobanovnormalisierten Vokalraum aller Versuchspersonen in GECO-FP mit schattierten Datenellipsen für die Referenzvokale (beschreiben zur besseren Sichtbarkeit $80 \%$ aller Messwerte). 
Die Vokalqualität für vokalische Füllpartikeln bewegt sich hauptsächlich im Bereich von $[œ e$ ə] und $[\varepsilon]$. Die Vokale vokalisch-nasaler Füllpartikeln überlappen mit den Bereichen von [œ $\bullet$ ə] und [a]. Im untermittelhohen Bereich zwischen $[\varepsilon]$ und [a] könnte die Vokalqualität des im Deutschen nicht phonologisch vorhandenen [æ] getroffen werden. Als Maß für die Vokalüberlappung berechne ich den Pillai-Wert (vgl. Abschnitt 4.4.3). Je höher der Pillai-Wert, desto größer ist die Distanz von $\mathrm{F}_{1}, \mathrm{~F}_{2}$ und $\mathrm{F}_{3}$ zwischen zwei Vokalen. Der in einer MANOVA ermittelte Pillai-Wert mit $F_{1}, F_{2}$ und $F_{3}$ als abhängiger Variable für die Distribution der Vokale in Vund VN-Formen in Abbildung 5.10 beträgt 0,1 ( $\mathrm{Df}=398, \mathrm{~F}=14,23, \mathrm{p}<0,001$, $\left.\eta^{2}=0,1\right)$. Die Vokale beider Formen überlappen sich also zum größten Teil $\left(\mathrm{F}_{3}\right.$ als Maß für die Lippenrundung kann in der zweidimensionalen Graphik nicht dargestellt werden, wird allerdings weiter unten nochmals diskutiert, vgl. Abbildung 5.13). Abbildung 5.11 zeigt, dass bei der Interpretation des errechneten Pillai-Wertes für alle Füllpartikeln in Abbildung 5.10 Zurückhaltung geboten ist, da sich die einzelnen Versuchspersonen sehr unterschiedlich verhalten, was in einer MANOVA nicht modelliert werden kann. Die Effektstärke $\eta^{2}$ liegt jedoch durchaus im Bereich eines mittelgroßen Effektes. ${ }^{115}$

$\rightarrow V \simeq V N$

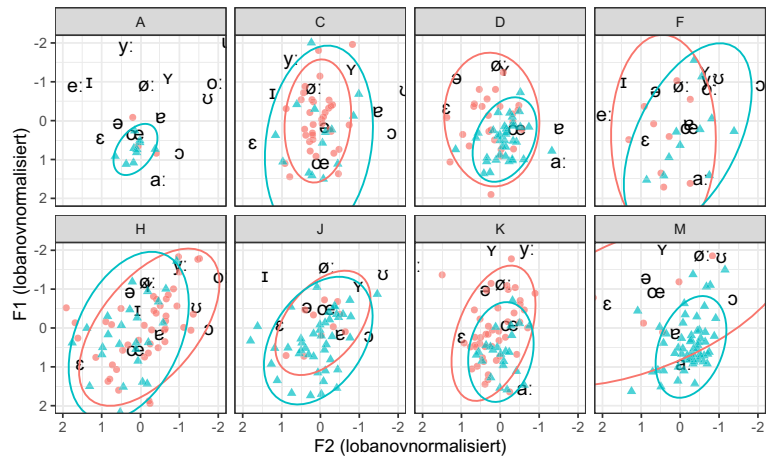

Abbildung 5.11: Vokalische (rot) und vokalisch-nasale (blau) Füllpartikeln in GECO-FP je Sprecherin in lobanovnormalisierten Vokalräumen (mittige Messung im Vokal, gezoomt). Datenellipsen umspannen $95 \%$ der Messpunkte.

\footnotetext{
${ }^{115}$ Zur Orientierung kann man von einem kleinen Effekt bei $\eta^{2}=0,01$, von einem mittleren Effekt bei $\eta^{2}=0,06$ und von einem großen Effekt bei $\eta^{2}=0,14$ sprechen (Cohen 1988: 188 f.).
} 
Tabelle 5.10 enthält die Pillai-Werte der V- und VN-Füllpartikeln aller acht Sprecherinnen in GECO-FP zum 50\%-Messpunkt Höhere Pillai-Werte beschreiben eine größere Distanz zwischen zwei Vokalverteilungen. Demnach unterscheiden Sprecherin $\mathrm{M}$ und $\mathrm{F}$ die Vokale in beiden Formen deutlicher als D und K. Für J, C, H und A lässt sich keine Aussage treffen, da ihre Modelle das Signifikanzniveau nicht erreichen.

Tabelle 5.10: Freiheitsgrade (Df), Pillai-, F- und p-Werte für die Vergleiche der Vokaldistribution in V- und VN-Formen je Versuchsperson (VP) in GECO-FP mittels einer multivariaten Varianzanalyse mit $\mathrm{F}_{1}, \mathrm{~F}_{2}$ und $\mathrm{F}_{3}$ als abhängiger Variable, sortiert nach Pillai-Werten.

\begin{tabular}{rr|rrr}
\hline VP & Df & Pillai & $\mathrm{F}$ & $\mathrm{p}$ \\
\hline $\mathrm{F}$ & 11 & 0,68 & 6,3 & $<0,05$ \\
$\mathrm{M}$ & 39 & 0,59 & 18,1 & $<0,001$ \\
$\mathrm{~A}$ & 9 & 0,29 & 1,0 & 0,456 \\
$\mathrm{D}$ & 34 & 0,29 & 4,4 & $<0,05$ \\
$\mathrm{~K}$ & 51 & 0,16 & 3,1 & $<0,05$ \\
$\mathrm{~J}$ & 39 & 0,16 & 2,4 & 0,087 \\
$\mathrm{C}$ & 43 & 0,09 & 1,4 & 0,255 \\
$\mathrm{H}$ & 57 & 0,05 & 1,0 & 0,417 \\
\hline
\end{tabular}

Die tiefere Realisierung des ersten Formanten in VN-Formen bei Versuchsperson $\mathrm{M}$ in Abbildung 5.11 und auch der Gesamteindruck in Abbildung 5.10 wirft die Frage auf, ob $F_{1}$ möglicherweise durch den nachfolgenden Nasal in VN-Formen abgesenkt wird. Allerdings gibt es für eine Absenkung von $\mathrm{F}_{1}$ in der Umgebung von Nasalen in einer Studie zum Englischen und über alle Vokale betrachtet keine Evidenz (Styler 2017: 2476). Betrachtet man die Formantkonturen aller Versuchspersonen für V- und VN-Formen in Abbildung 5.12, so ist auch hier zum Zeitpunkt des Messpunkts in der Mitte des Vokals (durchgezogene vertikale Linie) keine auffällige Koartikulation zu beobachten.

Zur Absicherung werden nochmals Messpunkte am Zeitpunkt des ersten Drittels des Vokals entnommen (vertikale gestrichelte Linie). Die Pillai-Werte für beide Zeitpunke je Versuchsperson weichen jedoch nicht signifikant voneinander ab (ungerichteter Paired t-test, $t=0,3, d f=7, p=0,8)$.

Der dritte Formant $\mathrm{F}_{3}$ ist ein Maß für die Lippenrundung; je tiefer $\mathrm{F}_{3}$, desto stärker ist die Lippenrundung (Stevens 1997: 474). Abbildung 5.13 gibt die $\mathrm{F}_{3}$-Werte der Füllpartikeln wieder und vergleicht sie mit ausgewählten Referenzvokalen aller acht Sprecherinnen in GECO-FP. Die Auswahl erfolgte anhand der Überschneidung 


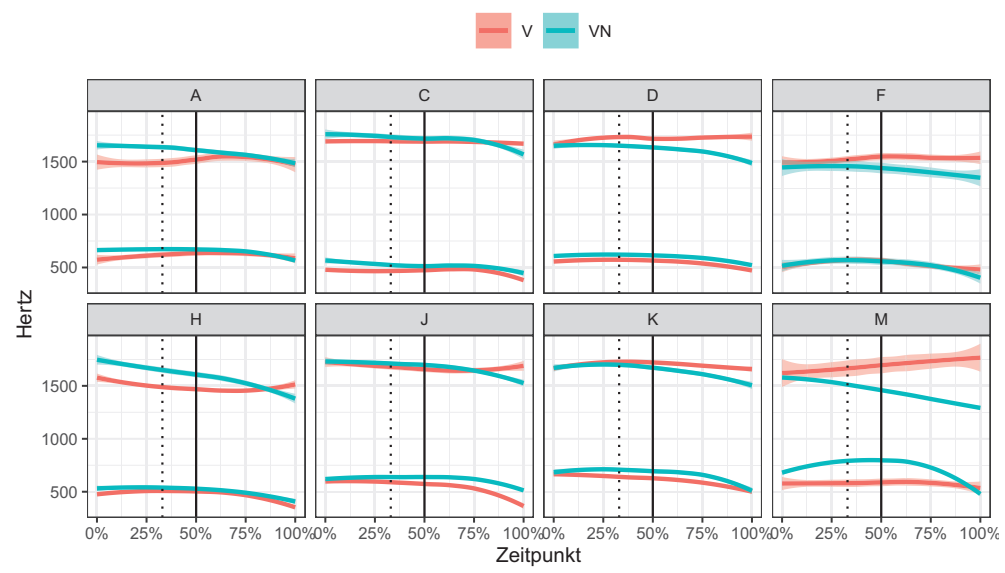

Abbildung 5.12: Gemittelte Formanttrajektorien von $F_{1}$ (unter $1000 \mathrm{~Hz}$ ) und $F_{2}$ (über $1000 \mathrm{~Hz}$ ) für den Vokal in V- (rot) und VN-Füllpartikeln (blau) je Versuchsperson in GECO-FP mit Zeitpunkten für mittige Messung der Formanten zum $50 \%$-Zeitpunkt (vertikale durchgezogene Linie) und zum $33 \%$-Zeitpunkt (vertikale gepunktete Linie).

der Füllpartikel- mit den Referenzvokalen in Abbildung 5.10. $\mathrm{F}_{3}$ zeigt besondere Ähnlichkeit mit [e] und [a:], wobei außerhalb der ersten Standardabweichung auch gerundetere Werte (Ähnlichkeit mit [œ]) und ungerundetere Werte (Ähnlichkeit mit $[ə]$ und $[\varepsilon])$ auftreten.

Die Vokaldauer für vokalische Füllpartikeln ist signifikant länger als für vokalischnasale Füllpartikeln (vgl. Abschnitt 5.2.1.1 sowie Abschnitt 6.1.3 in der konfirmatorischen Studie zur Erklärung mittels Ausgleichskürzung). Es ist daher möglich, dass sich die Vokaldauer auch auf die Formantwerte auswirkt. Abbildung 5.14 inspiziert nun den Zusammenhang zwischen Vokaldauer und den ersten beiden Formanten. Für $\mathrm{F}_{1}$ in Abbildung 5.14 a visualisieren zwei getrennte lineare Regressionslinien die verschiedenen Korrelationen zwischen dem ersten Formanten und Vokallängen unter und über $100 \mathrm{~ms}$. Für $\mathrm{F}_{1}$ steigt die Regressionslinie unter $100 \mathrm{~ms}$ stark an, ist allerdings für vokalische Füllpartikelvokale nicht signifikant (gerichtete Pearson's product-moment correlation, $r=0,27, t=1,08, D f=15, p=0,1$ ), für Vokale in vokalisch-nasalen Füllpartikeln hingegen durchaus (gerichtete Spe- 


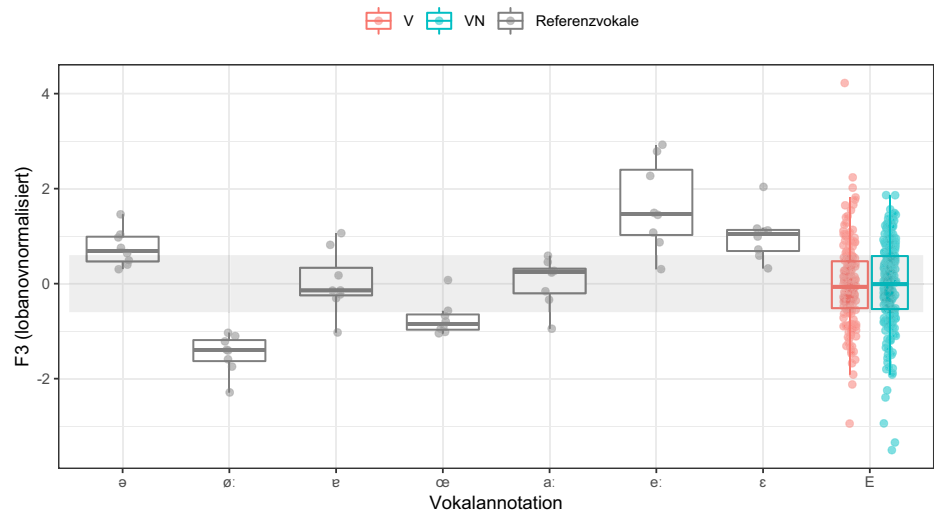

Abbildung 5.13: $\mathrm{F}_{3}$-Werte der Vokale in vokalischen (rot) und vokalisch-nasalen (grün) Füllpartikeln in GECO-FP für ausgewählte Referenzvokale (mittige Messung im Vokal). Der graumarkierte Bereich kennzeichnet eine Standardabweichung um den $\mathrm{F}_{3}$-Mittelwert der Füllpartikelvokale.

arman's rank correlation rho, $\rho=0,4, S=59047, p<0,001) .{ }^{116}$ Über $100 \mathrm{~ms}$ ist die Korrelation zwischen $F_{1}$ und Vokaldauer sehr klein, erreicht jedoch kein Signifikanzniveau (für Vokale in V: gerichtete Spearman's rank correlation rho, $\rho=-0,03, S=715605, p=0,7$; für Vokale in VN: gerichtete Spearman's rank correlation rho, $\rho=0,05, S=413954, p=0,5) .{ }^{117}$ Möglicherweise erreichen die $\mathrm{F}_{1}$-Werte der Vokale in VN-Formen unter $100 \mathrm{~ms}$ aufgrund ihrer Kürze nicht ihr eigentliches Ziel, was einen sogenannten target undershoot darstellt.

Für $\mathrm{F}_{2}$ ergeben sich keine visuellen Anzeichen für eine Zweiteilung der Regressionsgeraden. Für V-Formen ergibt sich eine negative Korrelation zwischen der Vokaldauer und der $\mathrm{F}_{2}$-Höhe, d. h. je länger der Vokal ist, desto niedriger (und damit posteriorer) wird er (gerichtete Spearman's rank correlation rho, $\rho=-0,31, S=$ $1228567, p<0,001)$. Zwischen $\mathrm{F}_{2}$ und Vokaldauer in VN-Formen besteht nur ein niedriger positiver, nicht-signifikanter Zusammenhang (ungerichtete Spearman's

\footnotetext{
${ }^{116}$ Aufgrund der nicht normalverteilten vokalisch-nasalen Vokaldauern unter 100 ms (ShapiroWilk normality test, $W=0,95, p<0,01)$ wird eine Spearman-Korrelation verwendet.

${ }^{117}$ Aufgrund der nicht normalverteilten V- Vokaldauern über $100 \mathrm{~ms}$ (Shapiro-Wilk normality test, $W=0,94, p<0,001$ ) sowie der nicht normalverteilten VN-Vokaldauern über $100 \mathrm{~ms}$ (Shapiro-Wilk normality test, $W=0,96, p<0,001)$ wird in beiden Fällen eine Spearman-Korrelation verwendet.
} 
rank correlation rho, $\rho=0,09, S=1653958, p=0,2)$.
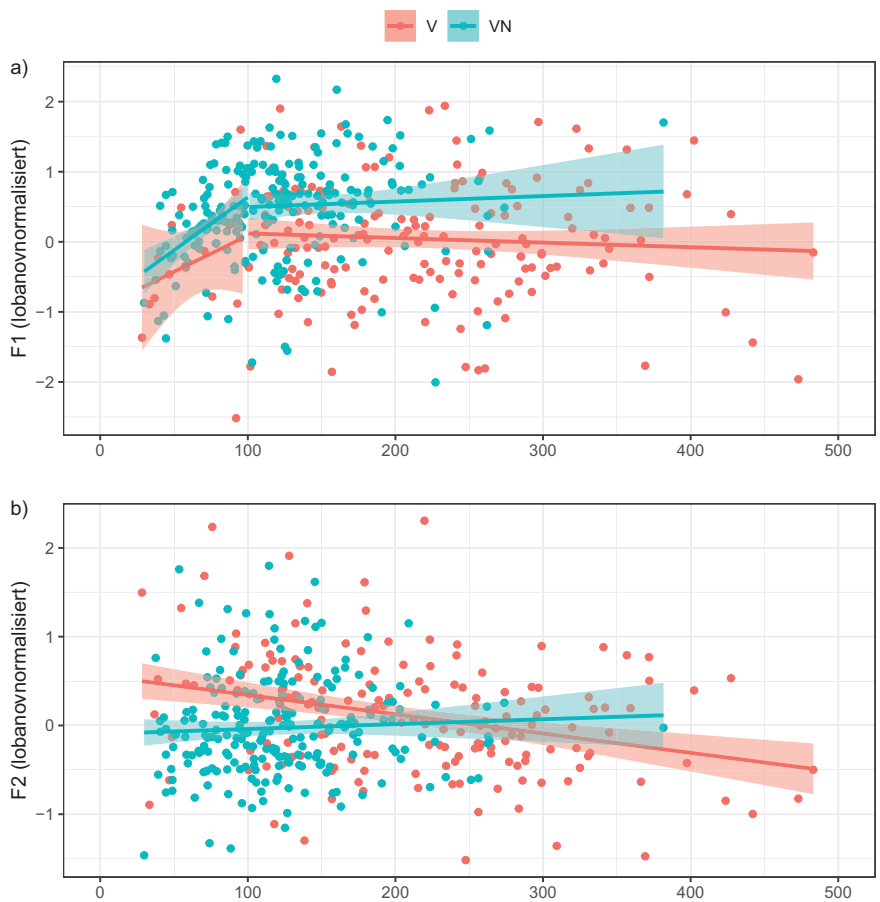

Abbildung 5.14: Zusammenhang zwischen $\mathrm{F}_{1}$ in (a) beziehungsweise $\mathrm{F}_{2}$ in (b) und Vokaldauer für vokalische (rot) und vokalisch-nasale (blau) Füllpartikeln. Jeweils drei Datenpunkte über $500 \mathrm{~ms}$ sind nicht abgebildet, aber in der Rechnung enthalten. Lineare Regressionen in a) sind separat berechnet für Werte unter und über $100 \mathrm{~ms}$.

Nachdem sich also Füllpartikelvokallängen unter $100 \mathrm{~ms}$ noch stark auf die Position des Formanten auswirken und möglicherweise ein target undershoot vorliegt, liegt es nahe, den Formantraum über alle Füllpartikeln nochmal zweigeteilt darzustellen - jeweils für Vokale unter und über 100 ms Länge. Abbildung 5.15 a veranschaulicht, dass sich die Variabilität für Dauern unter $100 \mathrm{~ms}$ besonders für VFüllpartikeln stark erhöht, mit einem erhöhten Pillai-Wert als Indikator für die abweichenden Distributionen im Vokalraum und einer großen Effektstärke (Pillai 
$\left.=0,25, d f=99, F=11, p<0,001, \eta^{2}=0,25\right)$. Dies mag daran liegen, dass ihr direkter segmentaler Kontext variabler als der von VN-Formen ist, die im rechten Segment immer den Nasal enthalten und somit ein anschließendes akustisches Ziel vorgeben. Für die Vokaldauern über $100 \mathrm{~ms}$ in Abbildung $5.15 \mathrm{~b}$ ändert sich kaum etwas im Vergleich zur Gesamtschau in Abbildung 5.10 - sowohl der PillaiWert als auch die Effektstärke $\eta^{2}$ bleiben auf dem gleichen Niveau (Pillai $=0,11$, $\left.d f=297, F=12,1, p<0,001, \eta^{2}=0,11\right)$.

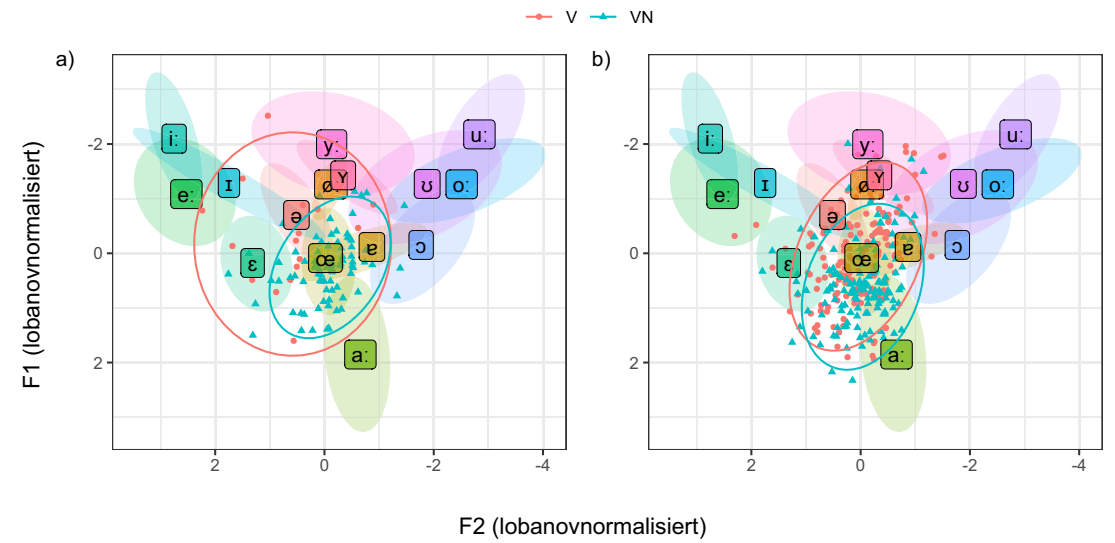

Abbildung 5.15: Lobanovnormalisierter Vokalraum aller Versuchspersonen in GECO-FP mit Datenellipsen für die Distributionen der Referenzvokale (Ellipsen enthalten zur besseren Sichtbarkeit $80 \%$ aller Referenzvokalmittelwerte) für Vokaldauern unter $100 \mathrm{~ms}$ (a) und über 100 ms (b). Vokalische Füllpartikeln sind mit roten Punkten, vokalisch-nasale Füllpartikeln mit blauen Dreiecken gekennzeichnet (mittige Messung im Vokal). Die entsprechenden Datenellipsen kennzeichnen $95 \%$ aller Messwerte.

Zur Quantifizierung der Überlappung zwischen V- und VN-Formantwerten wird für jeden Formanten ein lineares gemischtes Modell in Abhängigkeit von der Formausprägung der Füllpartikeln und der logarithmisierten Dauer gerechnet. Versuchspersonen gehen als Random Intercept, die unkorrelierte Vokaldauer der Füllpartikel als Random Slopes in das Modell ein. Interaktionen verbessern das Modell nicht. Das Modell ist in $\mathrm{R}$ wie folgt spezifiziert:

$1 \mid \operatorname{lmer}(\mathrm{F} \sim$ Form $+\log ($ Dauer $)+(1 \mid \mathrm{VP})+(0+\log ($ Dauer $) \mid \mathrm{vp})$, data $=\ldots$

Abbildung 5.16 zeigt die jeweiligen Effekte für jeden Formanten. Dort und in Tabel- 
Tabelle 5.11: Linear gemischte Modelle für $\mathrm{F}_{1}, \mathrm{~F}_{2}$ und $\mathrm{F}_{3}$ in Abhängigkeit von Form + $\log ($ Dauer $)+(1 / V P)+(0+\log ($ Dauer $) / V P)$, mit erklärter Varianz $R^{2}$.

\begin{tabular}{lrrr}
\hline & $\mathrm{F} 1$ & \multicolumn{1}{c}{$\mathrm{F} 2$} & \multicolumn{1}{c}{$\mathrm{F} 3$} \\
\hline (Intercept) & $353,8(41,3)^{* * *}$ & $1767,1(81,4)^{* * *}$ & $2688,1(87,9)^{* * *}$ \\
FormVN & $65,6(9,2)^{* * *}$ & $-28,9(17,9)$ & $8,2(19,3)$ \\
$\log ($ Dauer $)$ & $45,7(10,0)^{* * *}$ & $-28,8(17,0)$ & $-2,0(17,9)$ \\
\hline AIC & 4253,2 & 5025,8 & 4994,1 \\
Num. obs. & 372 & 393 & 386 \\
Num. groups: vp & 8 & 8 & 8 \\
\hline $\mathrm{R}_{m}^{2} / \mathrm{R}_{c}^{2}$ & $0,07 / 0,62$ & $0,01 / 0,32$ & $0 / 0,24$ \\
\hline${ }^{* * *} p<0,001,{ }^{* *} p<0,01,{ }^{*} p<0,05$ & &
\end{tabular}

le 5.11 wird deutlich, dass nur der erste Formant signifkante Unterschiede zwischen V- und VN-Formen und ihrer Dauer zeigt: In VN-Formen ist er ca. $66 \mathrm{~Hz}$ höher als in V-Formen (d. h. er liegt tiefer im Vokalraum) und steigt für längere Vokaldauern weiter an (bzw. bewegt sich im Vokalraum nach unten).


Abbildung 5.16: Effekte für a) $F_{1}$, b) $F_{2}$ und c) $F_{3}$ des mittig gemessenen Vokals in Vund VN-Formen mit Form als Prädiktor.

\subsubsection{Phonationsart}

Abbildung 5.17 stellt die Verteilung der Vokale in V- und VN-Formen in GECO-FP nach Phonationsart dar. Nasale wurden nicht annotiert. VN-Formen zeigen keine laryngalisierten Vokale (von Laryngalisierung spreche ich bei phrasenfinaler Glottalisierung, vgl. Abschnitt 2.2.5). Insgesamt sind 13,9\% $(\mathrm{n}=55)$ aller $\mathrm{V}$ - und VNFüllpartikeln glottalisiert. Von den V-Formen sind es 20,3\%, von den VN-Formen $8,6 \%$. 


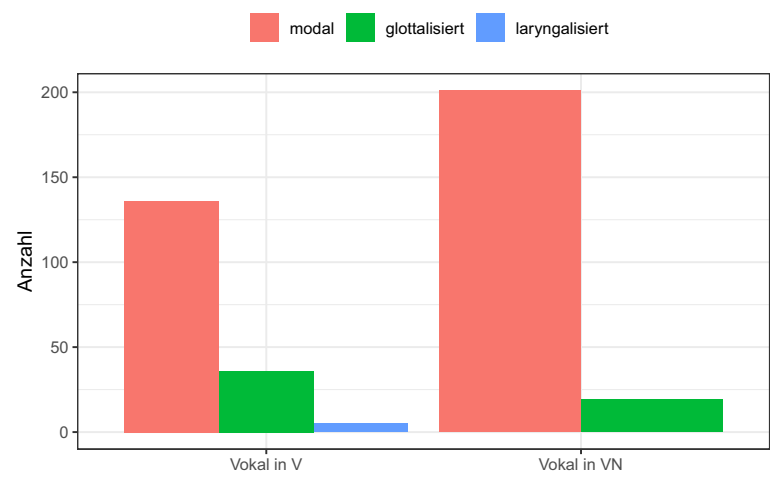

Abbildung 5.17: Anzahl der Vokale in V- und VN-Formen nach Phonationsart in GECO-FP.

Die Vokaldauern je Füllpartikelform sind in Abbildung 5.18 dargestellt, mit den genauen Werten für normalisierte und nichtnormalisierte Dauer in Tabelle 5.12. Modale Vokale in V-Formen sind nach paarweisen Vergleichen mithilfe von t-Tests mit gepoolter Standardabweichung und bonferronikorrigiertem p-Wert signifikant länger als glottalisierte $(p<0,05)$ und laryngalisierte Vokale $(p<0,05)$; glottalisierte und laryngalisierte Vokaldauern in V-Formen sind nicht signifikant unterschiedlich, was wohl daran liegt, dass insgesamt nur 5 laryngalisierte Vokale vorkommen. Auch modale Vokale in VN-Formen sind signifikant länger als glottalisierte Vokale (ungerichteter Welch Two Sample t-test, $\left.t=4, d f=31,3, p<0,001, K I_{95 \%}=[0,11 ; 0,33]\right)$. Zusammenfassend sind modale Vokale in Füllpartikeln also länger als glottalisierte Vokale.

Tabelle 5.12: Mittelwert und Standardabweichung für nichtnormalisierte Dauer (in ms) und normalisierte Dauer (in Silben $\sigma$ ) für verschiedene Vokalphonationen je Füllpartikelform in GECO-FP.

\begin{tabular}{ll|rr|rr}
\hline Form & Phonationsart & $\bar{x}(\mathrm{~ms})$ & $\mathrm{s}$ & $\bar{x}(\sigma)$ & $\mathrm{s}$ \\
\hline Vokal in V & modal & 219,0 & 96,3 & 1,2 & 0,6 \\
Vokal in V & glottalisiert & 174,4 & 83,1 & 1,0 & 0,5 \\
Vokal in V & laryngalisiert & 94,8 & 29,8 & 0,5 & 0,2 \\
Vokal in VN & modal & 126,6 & 66,7 & 0,7 & 0,4 \\
Vokal in VN & glottalisiert & 92,5 & 37,8 & 0,5 & 0,2 \\
\hline
\end{tabular}




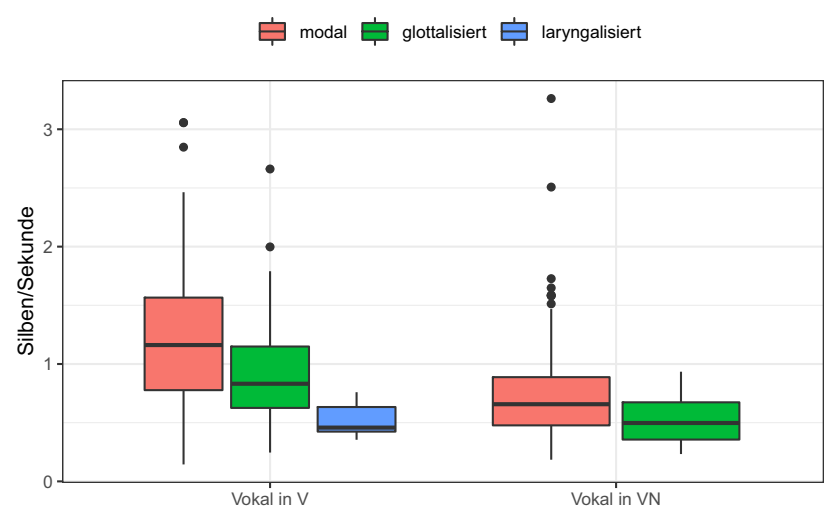

Abbildung 5.18: Normalisierte Dauer der Vokale in V- und VN-Formen nach Phonationsart in GECO-FP.

Das Histogramm in Abbildung 5.19 zeigt die Häufigkeit der Grundfrequenz über die Anzahl der modalen und glottalisierten Füllpartikeln. In 32 Fällen misst der Algorithmus $0 \mathrm{~Hz}$. Die restlichen Daten zerfallen in zwei Gruppen (was aus Abschnitt 5.1.4 schon bekannt ist, vgl. Abbildung 5.7), eine erste zwischen 0 und $140 \mathrm{~Hz}$ (der Mittelwert dieser Häufung beträgt 98,1 Hz) und eine zweite ab $140 \mathrm{~Hz}$ (der Mittelwert beträgt 203,5 Hz). In Abschnitt 5.1.4 wurde gemutmaßt, dass die Daten in der ersten Gruppe möglicherweise auf glottalisierte Formen zurückzuführen seien dieser Eindruck wird nun durch die Betrachtung der Phonationsartannotation bestätigt. 


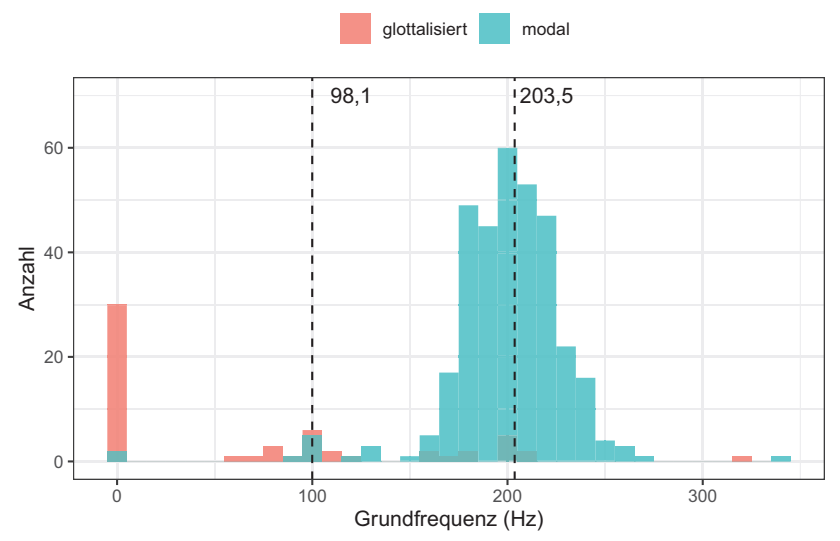

Abbildung 5.19: Phonationsarthistogramm der im Mittelpunkt aller nicht-glottalen Füllpartikeln gemessenen Grundfrequenz mit einer Klassenbreite von $10 \mathrm{~Hz}$ und den Mittelwerten der beiden Häufungen (gestrichelte Linien) über alle Formen je Häufung in GECO-FP.

\subsection{Form und Kontext}

\subsubsection{Sequenzielle Kontexte}

Sequenzielle Kontexte können auf Wort- oder Segmentebene analysiert werden. Zur Betrachtung und dem Vergleich der Wort-Pause-Kontexte rekategorisiere ich die sequenziellen Kontexte der hes-Ebene in Anlehnung an Leeuw (2007) in Wort- und Pausenkontexte (unabhängig von ihrer Art). Tabelle 5.13 enthält die Distribution der nicht-glottalen Füllpartikelformen in Wort-Pause-Kontexten für V-, VN- und N-Formen (orthographisch repräsentiert als $\ddot{a}$, ähm und $h m$ ) im Vergleich zu den deutschsprachigen Interviewdaten von Leeuw (vgl. Abschnitt 3.2.2.2). ${ }^{118}$

Obwohl die Interviewdaten von Leeuw (ebd.) nur unter Vorbehalt mit den hier analysierten spontansprachlichen Dialogen vergleichbar sind, ist doch auffällig, dass der Wort-FP-Wort-Kontext hier ähnliche proportionale Verteilungen für Füllpartikelformen zeigt. In den spontansprachlichen Dialogen scheint es in Wort-FP-Wortund Pause-FP-Wort-Kontexten eine leichte Formpräferenz für äh zu geben, während

\footnotetext{
${ }^{118}$ Diese Einteilung enthält die auf der Ebene hes annotierten Kontexte (vgl. im Anhang Tabelle A.2) und ordnet sie den Kategorien ,Wort' (as/ps) oder ,Pause' (ap/pp, ah/ph, at/pt) zu (ohne Clicks, extralinguistische Entitäten und ohne Zweifelsfälle).
} 
Tabelle 5.13: Prozentuale Anteile vokalischer, vokalisch-nasaler und nasaler Füllpartikeln an Wort-Pause-Kontexten in GECO-FP mit Anzahl (n) und bei Leeuw (2007) mit p-Wert und Teststatistik für ihre Unterschiede je Zeile (p für $\chi^{2}$-Test basierend auf 2000 Simulationen).

\begin{tabular}{|c|c|c|c|c|c|c|c|c|c|c|c|}
\hline \multirow[b]{2}{*}{ Kontext } & \multirow[b]{2}{*}{$\mathrm{V}$} & \multicolumn{5}{|c|}{ GECO-FP } & \multicolumn{3}{|c|}{ Leeuw (2007) } & \multirow[b]{2}{*}{$\mathrm{p}$} & \multirow[b]{2}{*}{ Test } \\
\hline & & (n) & $\mathrm{VN}$ & $(\mathrm{n})$ & $\mathrm{N}$ & (n) & $\mathrm{V}$ & $\mathrm{VN}$ & $\mathrm{N}$ & & \\
\hline Wort-FP-Wort & 54 & $(73)$ & 46 & (61) & 0 & $(0)$ & 42 & 58 & 0 & $=0,12$ & Fisher \\
\hline Wort-FP-Pause & 33 & (28) & 66 & (56) & 1 & (1) & 17 & 82 & 1 & $<0,05$ & $\chi^{2}=6,9$ \\
\hline Pause-FP-Wort & 53 & $(40)$ & 41 & $(31)$ & 5 & (4) & 6 & 75 & 19 & $<0,001$ & $\chi^{2}=55,2$ \\
\hline Pause-FP-Pause & 12 & $(5)$ & 84 & $(36)$ & 5 & (2) & 27 & 58 & 15 & $<0,001$ & $\chi^{2}=16,2$ \\
\hline
\end{tabular}

nach einem Wort und vor einer Pause sowie interpausal eher $\ddot{a} h m$ präferiert wird.

Die Annotation der füllpartikeladjazenten Kontexte in dieser Arbeit ermöglicht nun einen detaillierten Einblick in die Verteilung der Formen je Kontext. Zu diesem Zweck betrachte ich die prominenten glottalen Formen ? und G und vergleiche sie mit nicht-glottalen vokalischen und vokalisch-nasalen Formen in den häufigsten direkten Kontexten ( $>$ 5) (vgl. Abbildung 5.20). Der häufigste Kontext as FP ps enthält

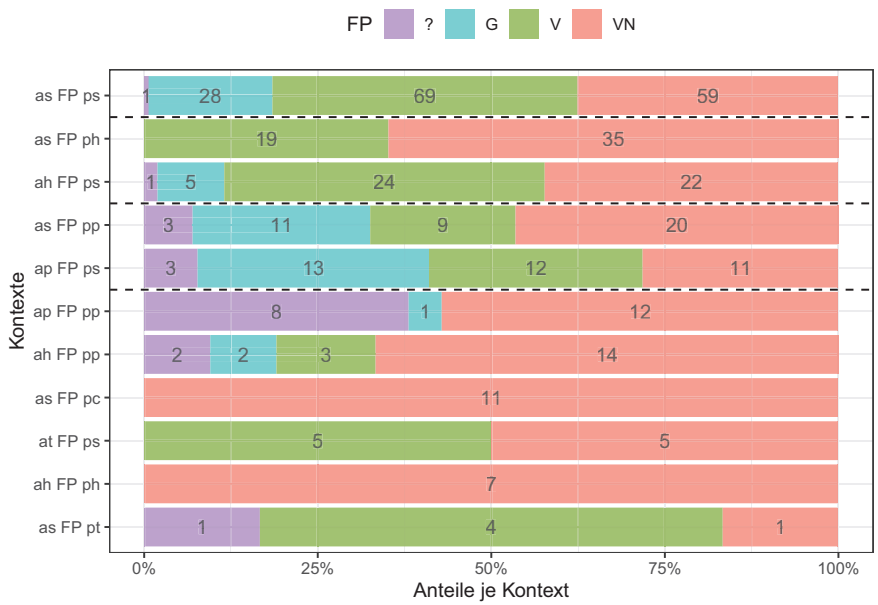

Abbildung 5.20: Anteil der V- und VN-Füllpartikelformen je Kontexte ( $\mathrm{n}>5)$ in GECO-FP (FP = Füllpartikel, Antezedentia: at = Turn, as = Segment, ap = Pause, ah $=$ Atmungspause; Postzedentia: $\mathrm{ps}=$ Segment, $\mathrm{pp}=$ Pause, $\mathrm{ph}=$ Atmungspause, $\mathrm{pc}=$ Click). Gestrichelte Linien gliedern die Graphik in ähnliche Kontexte. 
vor und nach der Füllpartikel ein Lautsegment; $15 \%$ der Füllpartikelformen entfallen auf die glottalen Formen ? und G, $36 \%$ auf die V und $28 \%$ auf die VN-Formen. Der zweit- und dritthäufigste Kontext ist komplementär zueinander; im zweiten (as FP $p h)$ folgt die Füllpartikel auf einen Laut und steht vor Atmung ( $31 \%$ V, $57 \%$ VN), im dritten ( $a$ h FP ps) erfolgt erst die Atmung, dann die Füllpartikel und anschließend wird ein Lautsegment geäußert $(38 \% \mathrm{~V}, 36 \% \mathrm{VN})$. Der vierte und fünfte Kontext beschreibt Segmente und Pausen vor und nach Füllpartikeln. Glottale Füllpartikeln sind im Kontext as FP pp proportional weniger häufig als in ap FP ps. In den ersten fünf Kontexten kommt die V-Form prozentual am häufigsten in as FP ps und ah FP ps vor, die VN-Form hingegen am häufigsten in as FP ph und as FP pp. In ap FP ps sind V- und VN-Formen gleich häufig, während glottale Füllpartikeln etwa ein Drittel dieses Kontextes ausmachen. Ab dem sechsten Kontext nehmen die enthaltenen absoluten Instanzen stark ab, weswegen ich hier von einer Interpretation absehe. Allerdings ist noch erwähnenswert, dass die VN-Form zwischen Pause, stillen Pausen oder Kombinationen davon immer mindestens $50 \%$ erreicht (in ap FP pp, ah FP $p p$ und ah FP $p h)$.

Hängt die Wahl einer Formvariante von ihrem sequenziellen Lautkontext ab? Abbildung 5.21 zeigt die Verteilung adjazenter Laute (in SAMPA) für den intersegmentalen Kontext as FP ps für V- und VN-Formen. 11 Kontexte kommen sowohl bei V-als auch bei VN-Formen vor, einige Treffer häufiger als einmal. Um zu ermitteln, ob die beiden Tableaus unabhängig voneinander sind, werden 10000 Permutationen des V-Tableaus simuliert und für jede Permutation die jeweils mit dem VN-Tableau übereinstimmenden Treffer gezählt. Anschließend ermittle ich mithilfe eines Binomialtests die Wahrscheinlichkeit, 11 oder mehr Übereinstimmungen in den 10000 Vergleichen beider Tableaus zu erhalten. Da dies in 284 von 10000 Fällen geglückt ist, ist $\mathrm{p}<0,01$ (der p-Wert entspricht hier der oberen Grenze des $95 \%$-Konfidenzintervalls). Die Hypothese, dass die beiden Tableaus unabhängig sind, muss somit abgelehnt werden. Dies bedeutet, dass es keine Evidenz dafür gibt, dass V-Formen einen anderen segmentalen Kontext haben als VN-Formen (sonst hätte es weniger gemeinsame Treffer geben müssen, nämlich maximal acht). Die Hypothese, dass die Wahl einer V- oder VN-Form von ihren direkt vorangehenden und nachfolgenden Lauten abhängt, muss demnach abgelehnt werden. 


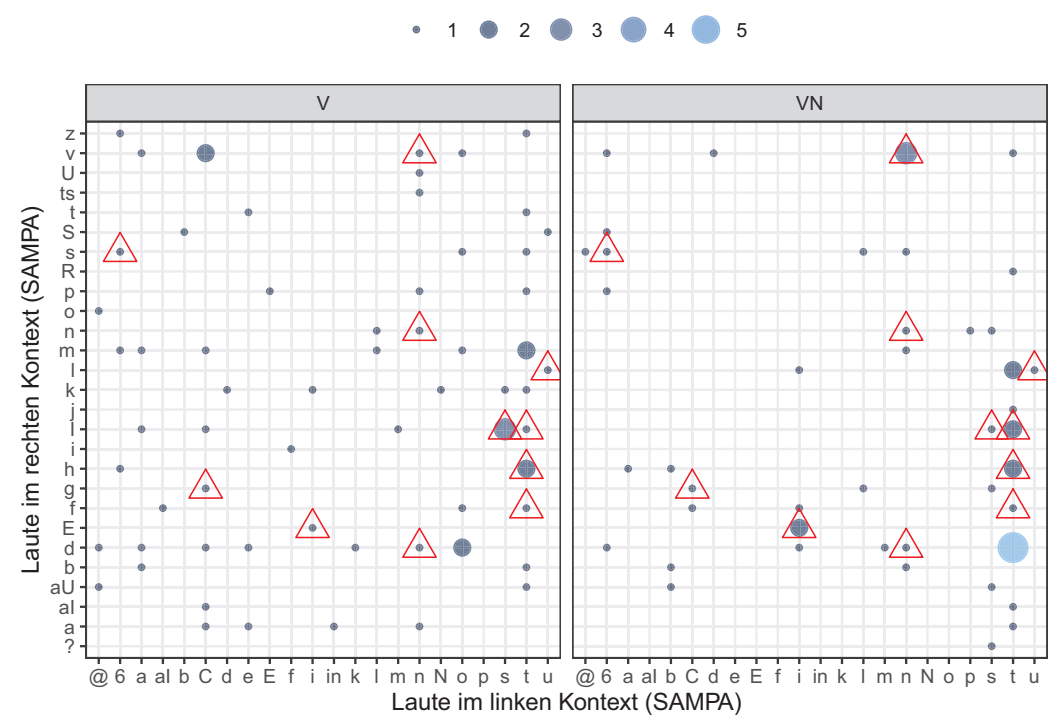

Abbildung 5.21: Anzahl und Verteilung der V- und VN-Füllpartikelformen je linkem und rechtem Kontext für alle Kontexte mit Segmenten ( $\mathrm{n}>5$ ) in GECO-FP (für SAMPA vgl. Tabelle D.1). Rote Dreiecke kennzeichnen identische Kontexte für V und VN.

\subsubsection{Dauer}

Über den Zusammenhang von Füllpartikeldauer und sequenzielle Kontexte gibt es bisher hauptsächlich Studien, welche die nachfolgenden oder vorangehenden Pausen untersuchen. Hier dehne ich den Skopus der Untersuchung weiter aus und frage zusätzlich, ob sequenzielle Kontexte einen Einfluss auf die Füllpartikeldauer haben. Die Ergebnisse eines linearen gemischten Modells mit Dauer als abhängiger Variable werden in Abbildung 5.22 und Tabelle 5.14 dargestellt. Als unabhängige Variablen gehen die Kontexte mit $n \geq 10$ Füllpartikeln in die Analyse ein (dabei muss jede Form mindestens einmal vorkommen), sowie die beiden Form V und VN ein, als zufällige Schnittpunkte die Versuchspersonen. Random slopes und Interaktionen verbessern das Modell nicht.

${ }_{1} \mid \operatorname{lmer}($ FP-Dauer $\sim$ Kontext + Form $+(1 \mid \mathrm{VP})$, data $=\ldots)$

Füllpartikeln zwischen Segment und Pause sind mit ca. 66,7 ms signifikant länger 

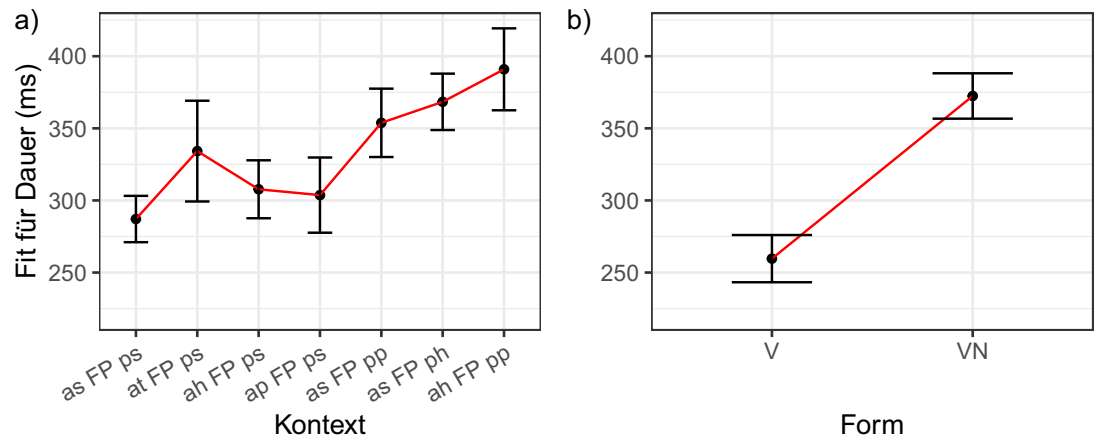

Form

Abbildung 5.22: Effekte der Füllpartikeldauern a) für sequenzielle Kontexte wie SegmentFP-Segment- (as FP ps), Turnpause-FP-Segment- (at FP ps), Atmung-FP-Segment- (ah FP $p s$ ), Pause-FP-Segment- (ap FP ps), Segment-FP-Pause- (as FP pp), Segment-FP-Atmung(as FP $p h$ ) sowie Atmung-FP-Pause-Kontexten ( ah FP $p p$ ) und b) für Form in GECO-FP.

als intersegmentale Füllpartikeln; ebenso sind Füllpartikeln zwischen einem Segment im linken und Atmung im rechten Kontext mit ca. 81,3 ms und Füllpartikeln zwischen Atmung und Pause mit 103,8 ms signifikant länger als intersegmentale Füllpartikeln. Zudem sind, wie schon bekannt, VN-Füllpartikeln mit ca. 112,8 ms signifikant länger als V-Füllpartikeln. Post-hoc-Tests mit Tukey-Korrektur ergeben keine weiteren signifikanten Unterschiede. Es stellt sich jedoch die Frage, ob die hier vorliegenden Effekte längerer Füllpartikeln vor Pausen und Atmungspausen mit Phrasengrenzen zusammenfallen.

$\mathrm{Zu}$ Pausenkontexten besteht in der Literatur mindestens seit der Studie von Clark \& Fox Tree (2002) die Annahme, dass die Länge der nachfolgenden stillen Pause von der Dauer der Füllpartikel abhängt. Abbildung 5.23 zeigt die Abhängigkeit der Länge der nachfolgenden Pause von der Länge der Füllpartikelform. Die normalisierte mittlere Dauer der nachfolgenden Pause nach V-Formen beträgt $\bar{x}=3 \sigma$ (sd $=$ $2,68)$ und nach VN-Formen $\bar{x}=2,45 \sigma(\mathrm{sd}=1,62)$. Die linearen Geraden zeigen für V-Formen einen schwachen negativen Zusammenhang $(\rho=-0,2)$, für VN-Formen einen schwachen positiven Zusammenhang $(\rho=0,1)$, beide jedoch nicht signifikant. Ein lineares gemischtes Modell mit der logarithmischen Füllpartikeldauer als abhängiger Variable, Form und Dauer der Füllpartikel als unabhängiger Variable und Versuchspersonen als Random Intercepts zeigt keine signifikanten Effekte. 
Tabelle 5.14: Linear gemischtes Modell für die Füllpartikeldauer in Abhängigkeit von Kontext + Form $+(1 / V P)$, mit erklärter Varianz $R^{2}$.

\begin{tabular}{lc}
\hline & Dauer \\
\hline Intercept) & $227,6(17,1)^{* * *}$ \\
Kontextat FP ps & $47,1(33,7)$ \\
Kontextah FP ps & $20,6(17,7)$ \\
Kontextap FP ps & $16,6(23,9)$ \\
Kontextas FP pp & $66,7(21,8)^{* *}$ \\
Kontextas FP ph & $81,3(16,9)^{* * *}$ \\
Kontextah FP pp & $103,8(27,0)^{* * *}$ \\
FormVN & $112,8(13,4)^{* * *}$ \\
\hline AIC & 3715,6 \\
Num. obs. & 311 \\
Num. groups: vp & 8 \\
\hline $\mathrm{R}_{m}^{2} / \mathrm{R}_{c}^{2}$ & $0,32 / 0,4$ \\
\hline${ }^{* * *} p<0,001,{ }^{* *} p<0,01,{ }^{*} p<0,05$
\end{tabular}

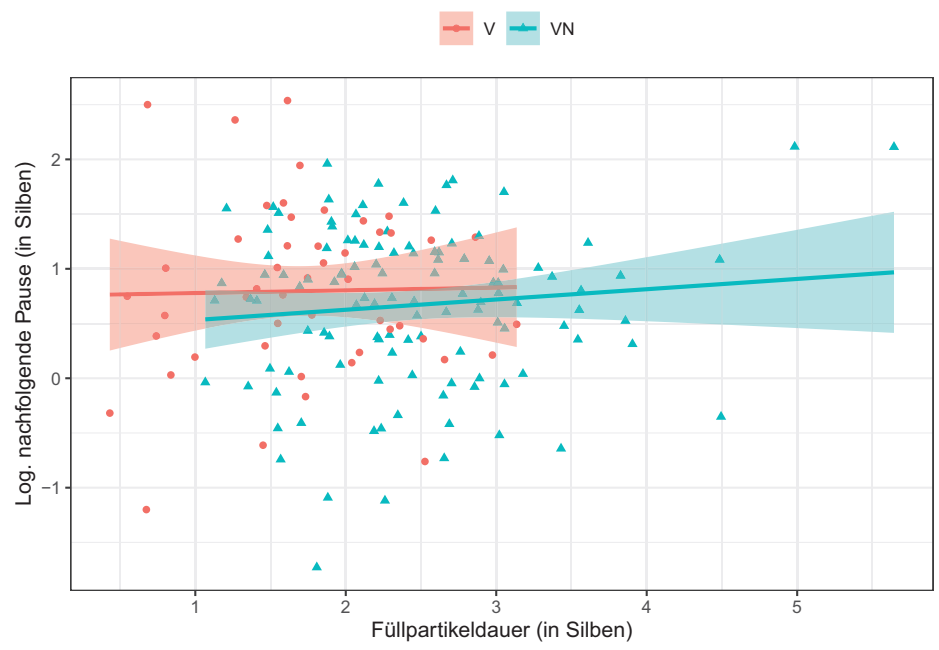

Abbildung 5.23: Füllpartikeldauer und Dauer der nachfolgenden Pause für V- und VNFüllpartikelformen $\left(\mathrm{n}_{V}=49, \mathrm{n}_{V N}=116\right)$ in GECO-FP. 
$\operatorname{lmer}(\log ($ Postpositionsdauer $) \sim$ Form + Formdauer $+(1 \mid \mathrm{VP})$, data $=\ldots)$

Damit können die Ergebnisse von Clark \& Fox Tree (2002) nicht repliziert werden. Auch das Maß der ,totalen Verzögerung، in Anlehnung an Rose (2015) (vgl. Abschnitt 3.2.2.1) führt zu keinem anderen Ergebnis. Es beträgt für die Dauer von V-Formen mit nachfolgender Pause $\bar{x}=4,73 \sigma / \mathrm{s}(\mathrm{sd}=2,69)$, für VN-Formen mit nachfolgender Pause $\bar{x}=4,9 \sigma / \mathrm{s}(\mathrm{sd}=1,92)$. Der Unterschied ist nicht signifikant (ungerichteter Welch Two Sample t-test, $t=-0,42, d f=69,54, p=0,68$, $\left.\mathrm{KI}_{95 \%}=[-1,02 ; 0,67]\right)$.

Die hier vorliegenden Ergebnisse zeigen also keine Abhängigkeit der nachfolgenden Pausenlänge von der Form oder Dauer einer Füllpartikel. Füllpartikeln sind jedoch sowohl interpausal als auch präpausal signifikant länger als intersegmental (vgl. Abbildung 5.22 und Tabelle 5.14).

\subsubsection{Grundfrequenz}

Die $f_{0}$ einer Füllpartikel niedriger als die mittlere $f_{0}$ einer Versuchsperson. Aber ist die Grundfrequenz nicht-glottaler Füllpartikeln auch von ihrem Kontext abhängig? Hierfür ziehe ich die im vorigen Abschnitt 5.2.1.1 betrachteten Kategorien heran. Für jede Füllpartikel $\left(n_{V}=179, n_{V N}=179\right)$ wird der Mittelwert gebildet. Werte unter $140 \mathrm{~Hz}$ (Messfehler und glottalisierte Phasen) und über $350 \mathrm{~Hz}$ sowie nichtnormalverteilte Residuen werden ausgeschlossen (insgesamt 1\%). Die Abhängigkeit der $\mathrm{f}_{0}$-Mittelwerte wird in einem linearen gemischten Modell berechnet, mit Form und Position als unabhängigen Faktoren sowie Random Intercepts für Versuchspersonen. Abbildung 5.24 visualisiert die Modellvorhersagen in Tabelle 5.15.

$1 \operatorname{lmer}(\mathrm{F} 0 \sim$ Form + Kontext $+(1 \mid \mathrm{VP})$, data $=\ldots)$

V- und VN-Füllpartikeln unterscheiden sich selbst nicht in ihrer Grundfrequenz. Ausgehend von Füllpartikeln in intersegmentaler Position (as FP ps) sind Füllpartikeln, denen einen Turnpause (die andere Person spricht) vorangeht und ein Segment nachfolgt ( at FP ps) signifikant höher $(20,3 \mathrm{~Hz})$. Selbiges gilt für Füllpartikeln, denen eine Atmungspause vorangeht und ein Segment nachfolgt (12,3 Hz höher). Der Aufbau dieser beiden Kontexte könnte auch der Beginn einer prosodischen Phrase sein, was die relativ hoch einsetzende $f_{0}$ erklären würde - sicher kann das jedoch nur in Kombination mit einer Intonationsphrasenannotation festgestellt werden (und wird dort auch bestätigt, vgl. Abschnitt 6.2.3.2). Umgekehrt gilt für die Kontexte as FP 

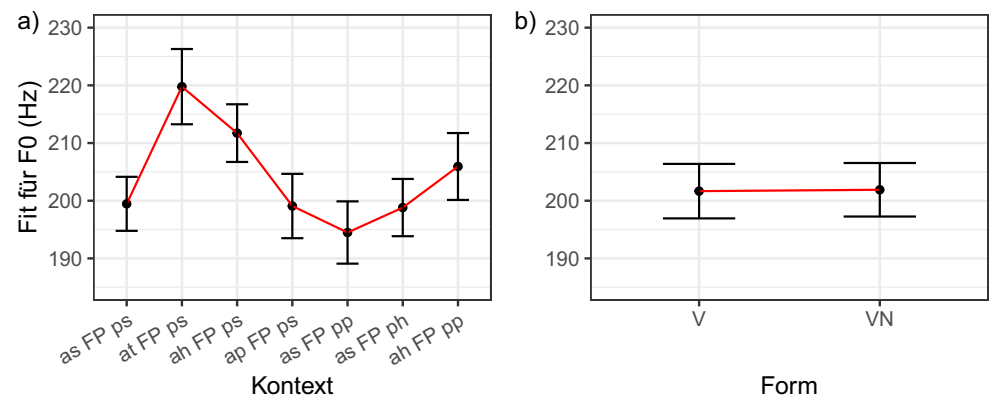

Form

Abbildung 5.24: Effekte der $\mathrm{f}_{0}$-Mittelwerte für a) sequenzielle Kontexten wie Segment-FPSegment- (as FP ps), Turnpause-FP-Segment- (at FP ps), Atmung-FP-Segment- (ah FP ps), Pause-FP-Segment- (ap FP ps), Segment-FP-Pause- (as FP pp), Segment-FP-Atmung- (as $F P p h$ ) sowie Atmung-FP-Pause-Kontexten ( $a h F P p p)$ und b) Form in GECO-FP.

$p p$ und as FP $p h$, die mit signifikant niedrigerer $\mathrm{f}_{0}$ geäußert werden als at FP ps und ah FP ps (vgl. Tabelle 5.16), dass hier einer prosodischen Phrase vorliegen könnte (auch dies ist später für die konfirmatorische Studie in Abbildung 6.33 zu sehen).

Tabelle 5.15: Modellvorhersagen für $\bar{x}_{f_{0}} \sim$ Form + Kontext $+(1 \mid \mathrm{VP})$, mit erklärter Varianz $R^{2}$.

\begin{tabular}{lc}
\hline & ${\text { Mittlere } \mathrm{f}_{0}}$ \\
\hline (Intercept) & $199,3(4,8)^{* * *}$ \\
FormVN & $0,2(2,0)$ \\
Kontextat FP ps & $20,3(5,0)^{* * *}$ \\
Kontextah FP ps & $12,3(2,6)^{* * *}$ \\
Kontextap FP ps & $-0,4(3,6)$ \\
Kontextas FP pp & $-5,0(3,3)$ \\
Kontextas FP ph & $-0,6(2,5)$ \\
Kontextah FP pp & $6,5(4,0)$ \\
\hline AIC & 2471,9 \\
Num. obs. & 300 \\
Num. groups: vp & 8 \\
\hline $\mathrm{R}_{m}^{2} / \mathrm{R}_{c}^{2}$ & $0,07 / 0,8$ \\
\hline${ }^{* * *} p<0,001,{ }^{* *} p<0,01,{ }^{*} p<0,05$
\end{tabular}

Dass Prosodie durchaus eine konfundierende Rolle spielen könnte, zeigt sich auch daran, dass Füllpartikeln in at FP ps- und ah FP ps-Kontexten signifikant höher produziert werden als in ap FP ps-Kontexten (vgl. Tabelle 5.16) - obwohl in allen 
drei Kontexten eine neue prosodische Phrase beginnen könnte, ist dies für solche Kontexte mit stiller Pause vor der Füllpartikel ( $a p$ FP $p s$ ) weniger wahrscheinlich, da sie eine niedrigere Grundfrequenz zeigen.

Tabelle 5.16: Signifikante Kontraste der Position für $\mathrm{f}_{0}$ (ohne Referenzniveau as FP ps), mit Schätzer $\beta$ und Standardabweichung ( $\mathrm{s}$ ) für $\mathrm{f}_{0}$, Freiheitsgraden (df), $95 \%$-Konfidenzintervall (KI) und t-Statistik, korrigiert für multiple Vergleiche nach Tukey.

\begin{tabular}{lrrrrrrr}
\hline Vergleich & $\beta$ & $\mathrm{s}$ & $\mathrm{df}$ & Unteres KI & Oberes KI & $\mathrm{t}$ & $\mathrm{p}$ \\
\hline at FP ps - ap FP ps & 20,7 & 5,9 & 286,6 & 3,27 & 38,11 & 3,53 & $<0,01$ \\
at FP ps - as FP pp & 25,3 & 5,6 & 286,0 & 8,60 & 41,97 & 4,50 & $<0,001$ \\
at FP ps - as FP ph & 21,0 & 5,2 & 285,8 & 5,64 & 36,28 & 4,06 & $<0,01$ \\
ah FP ps - ap FP ps & 12,6 & 3,9 & 286,3 & 0,93 & 24,34 & 3,21 & $<0,05$ \\
ah FP ps - as FP pp & 17,2 & 3,7 & 286,1 & 6,17 & 28,29 & 4,63 & $<0,001$ \\
ah FP ps - as FP ph & 12,9 & 3,1 & 286,6 & 3,82 & 22,00 & 4,22 & $<0,001$ \\
\hline
\end{tabular}

Im Rahmen dieser Analyse ist es demnach möglich, ein besseres Bild intersegmentaler Füllpartikeln zu erhalten. Hierzu extrahiere ich nochmals alle Füllpartikeln, die links und rechts jeweils zwei Wörter aufweisen (Fünfgramme). Hierbei ist natürlich nicht ausgeschlossen, dass die Wörter vor oder nach der Füllpartikel zu einer anderen Intonationsphrase zugeordnet sind. Da dies in GECO-FP nicht annotiert ist, kann dies für die hiesige Analyse nicht gelöst werden. Aus demselben Grund ist auch eine Fünfgram-Analyse von Pause-Füllpartikel-Wort oder Wort-Füllpartikel-Pause-Sequenzen nicht interpretierbar - dies gilt für solche Einheiten sogar noch eindringlicher, da Pausen oftmals Grenzen einer prosodischen Phrase markieren. Letztere Kontexte bleiben also vorerst außen vor.

Für jedes Wort in dem Fünfgram Wort-Wort-FP-Wort-Wort $\left(n_{V}=5 \cdot 73, n_{V N}=\right.$ $5 \cdot 61$ ) wird der Mittelwert gebildet. Werte unter $140 \mathrm{~Hz}$ (Messfehler und glottalisierte Phasen) und über $350 \mathrm{~Hz}$ sowie nichtnormalverteilte Residuen werden ausgeschlossen (insgesamt 19,3\%) Die Abhängigkeit der $\mathrm{f}_{0}$-Mittelwerte wird in einem linearen gemischten Modell berechnet, mit Form, Position und ihrer Interaktion als unabhängigen Faktoren sowie random intercepts für Versuchspersonen und einem Index, der für jedes Fünfgram gleich ist. Abbildung 5.25 visualisiert die Modellvorhersagen in Tabelle 5.17.

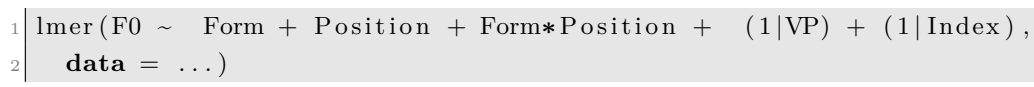




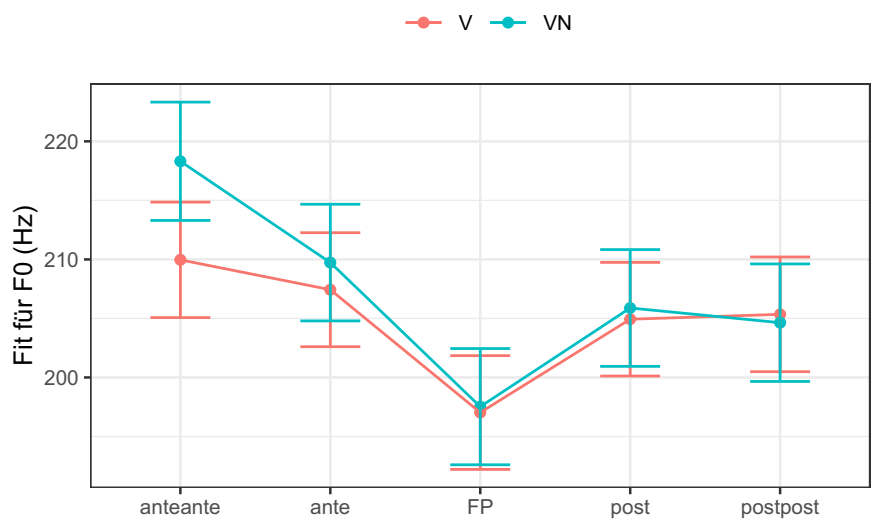

Abbildung 5.25: Modelleffekte für die $\mathrm{f}_{0}$-Mittelwerte zwei Wörter (anteante) und ein Wort (ante) vor sowie ein Wort (post) und zwei Wörter (postpost) nach nicht-glottalen Füllpartikeln (FP) in GECO-FP.

Tabelle 5.17: Modellvorhersagen für $\bar{x}_{f_{0}} \sim$ Form + Position + Form*Position + (1|VP) $+(1 \mid \mathrm{Id})$, mit erklärter Varianz $R^{2}$.

\begin{tabular}{lc}
\hline & Mittlere $\mathrm{f}_{0}$ \\
\hline (Intercept) & $210,0(4,9)^{* * *}$ \\
FormVN & $8,3(3,6)^{*}$ \\
Positionante & $-2,5(2,0)$ \\
PositionFP & $-12,9(1,9)^{* * *}$ \\
Positionpost & $-5,0(1,9)^{* *}$ \\
Positionpostpost & $-4,6(2,0)^{*}$ \\
FormVN:Positionante & $-6,0(2,8)^{*}$ \\
FormVN:PositionFP & $-7,9(2,8)^{* *}$ \\
FormVN:Positionpost & $-7,4(2,8)^{* *}$ \\
FormVN:Positionpostpost & $-9,1(2,9)^{* *}$ \\
\hline AIC & 4277,5 \\
Num. obs. & 541 \\
Num. groups: id & 134 \\
Num. groups: vp & 8 \\
\hline $\mathrm{R}_{m}^{2} / \mathrm{R}_{c}^{2}$ & $0,07 / 0,8$ \\
\hline${ }^{* * *} p<0,001,{ }^{* *} p<0,01,{ }^{*} p<0,05$ &
\end{tabular}


Das Modell zeigt für VN-Formen eine signifikante Abnahme von $8,6 \mathrm{~Hz}$ von anteante zu ante sowie von $12,2 \mathrm{~Hz}$ von ante zur Füllpartikel, wonach die Grundfrequenz zu den nächsten beiden Wörtern wieder zwischen $8,4 \mathrm{~Hz}$ (von $\mathrm{FP}$ zu post) und $7,1 \mathrm{~Hz}$ (von FP zu postpost) ansteigt, aber auf einem um $13,7 \mathrm{~Hz}$ niedrigeren Niveau als zum Zeitpunkt anteante verbleibt. Dies passt zur allgemein sinkenden $\mathrm{f}_{0}$-Deklination innerhalb einer prosodischen Phrase, was in der Diskussion weiter betrachtet wird. V-Formen zeigen eine signifikante Interaktion mit VN-Formen, nämlich ein um 8,3 Hz niedrigeres Ausgangsniveau zum Zeitpunkt anteante (vgl. Tabelle 5.17). In den anderen Positionen weicht die $\mathrm{V}-\mathrm{f}_{0}$ allerdings nicht signifikant von der $\mathrm{VN}-\mathrm{f}_{0} \mathrm{ab}$. Auch die $\mathrm{f}_{0} \mathrm{zu}$ den Zeitpunkten anteante und postpost ist für V-Formen nicht signifkant verschieden, d. h. für V-Formen kann nicht zwingend von einer generell absinkenden $\mathrm{f}_{0}$ über das Fünfgram hinweg ausgegangen werden, sondern es wird wieder ungefähr das Ausgangsniveau erreicht. Somit wurde gezeigt, dass Füllpartikeln in Fünfgrammen eine signifikant niedrigere $\mathrm{f}_{0}$ zeigen als ihr Kontext. Ohne Prosodieannotation bleibt die Analyse jedoch in Teilen Stipulation, da nicht auszuschließen ist, dass nach einer V-Füllpartikel zum Zeitpunkt ante oder anteante eine neue Intonationphrase beginnt und die $\mathrm{f}_{0}$ somit wieder ansteigt.

\subsubsection{Formanten}

Die Auswertung in Abschnitt 5.1.5 hat gezeigt, dass die Messpunkte in der Mitte der Füllpartikelvokale reliabel für die Bewertung der Vokalqualität sind. Hier überprüfe ich, ob die Vokalqualität zu diesem Messpunkt von den abstrahierten adjazenten Kontexten abhängig ist. Als erklärenden Faktor verwende ich wieder die sieben häufigsten Kontexte (vgl. Abschnitt 5.2.1) mit 315 Instanzen $\left(n_{V}=148, n_{V N}=167\right)$. Für $\mathrm{F}_{1}$ und $\mathrm{F}_{2}$ werden zwei separate gemischte lineare Modelle gerechnet, mit Kontext, Form und der logarithmisierten Dauer als Prädiktoren sowie Versuchspersonen als Random Intercepts und die unkorrelierte logarithmische Länge des Vokals als Random Slope je Versuchsperson. ${ }^{119}$ Interaktionen verbessern das Modell nicht. $3,8 \%$ der $\mathrm{F}_{1}$ - und 3,2\% der $\mathrm{F}_{2}$-Daten mussten aufgrund ihrer nichtnormalverteilten Residuen ausgeschlossen werden. Abbildung 5.26 zeigt die Effekte für $\mathrm{F}_{1}$ und $\mathrm{F}_{2}$, die zugehörigen Werte befinden sich in Tabelle 5.18.

\footnotetext{
${ }^{119} \mathrm{~F}_{3}$ hängt nicht von den direkten Kontexten ab, da ein Likelihood-Vergleich eines Modells mit Kontext $\left(F_{3} \sim\right.$ Kontext $+(0+\log ($ Dauer $\left.) / V P)\right)$ gegen ein Nullmodell $\left(F_{3} \sim 1+(0+\log (\right.$ Dauer $\left.) / V P)\right)$ nicht signifikant besser ist $(p=0,55)$.
} 

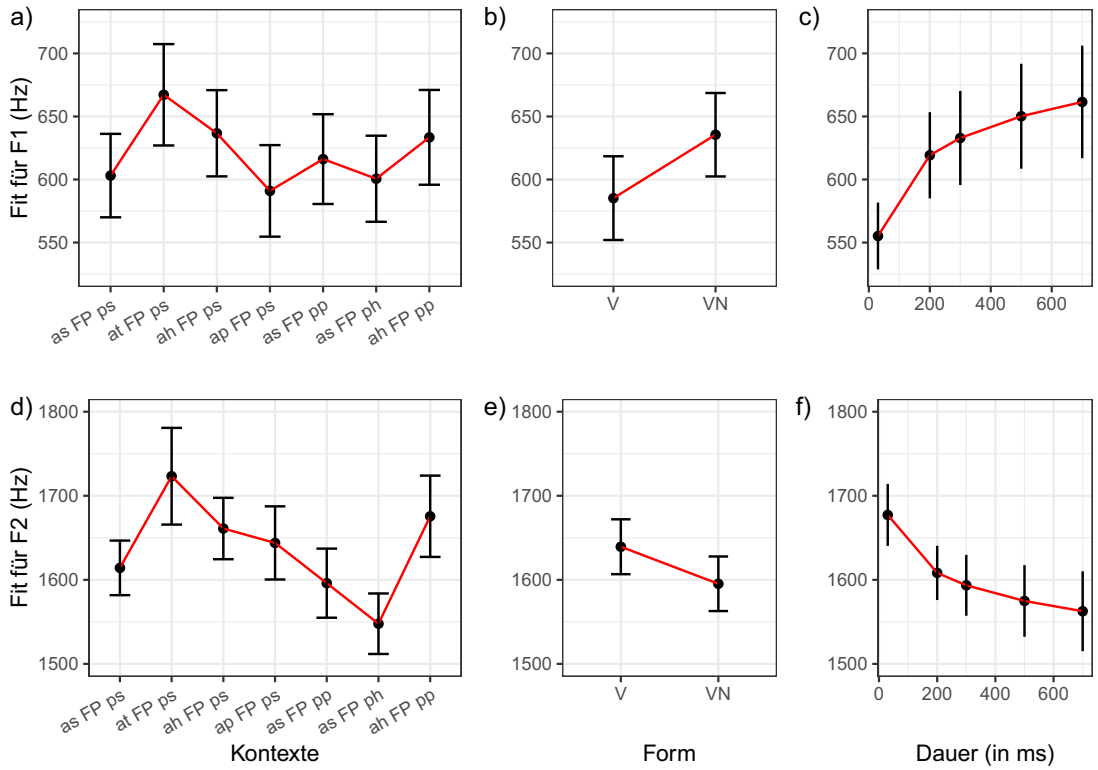

Abbildung 5.26: Modelleffekte für $\mathrm{F}_{1^{-}}$und $\mathrm{F}_{2}$-Werte in a)/d) adjazenten sequenziellen Kontexten für Segment-FP-Segment- (as FP ps), Turnpause-FP-Segment- (at FP ps), Atmung-FP-Segment- (ah FP ps), Pause-FP-Segment- (ap FP ps), Segment-FP-Pause- (as $F P p p$ ), Segment-FP-Atmung- (as FP $p h$ ) und Atmung-FP-Pause-Kontexte (ah FP $p p$ ), b)/e) für Form und c)/f) für Dauer in GECO-FP.

Ausgehend von Füllpartikeln in intersegmentaler Position (as FP ps) liegen die Füllpartikelvokale, denen einen Turnpause (die andere Person spricht) vorangeht und ein Segment nachfolgt (at FP ps) signifikant tiefer $(64,1 \mathrm{~Hz})$ und anteriorer $(109 \mathrm{~Hz})$ im Vokalraum. Füllpartikelvokale, denen eine Atmungspause vorangeht und ein Segment nachfolgt, sind auch signifikant tiefer $(33,6 \mathrm{~Hz})$ im Vokalraum als intersegmentale Füllpartikelvokale. Füllpartikelvokale zwischen Segment und Atmung (as FP $p h)$ sind signifikant anteriorer $(-66,5 \mathrm{~Hz})$ als intersegmentale Füllpartikelvokale. Post-hoc-Tests nach Tukey für Familien mit sieben Schätzungen (vgl. Tabelle 5.19) zeigen außerdem signifikante Unterschiede für $\mathrm{F}_{2}$ zwischen den gegensätzlichen Kontexten at FP ps und as FP ph $(175,5 \mathrm{~Hz})$ sowie zwischen ah FP ps und as FP ph $(113,3 \mathrm{~Hz})$. 
Tabelle 5.18: Linear gemischte Modelle für $\mathrm{F}_{1}$ und $\mathrm{F}_{2}$ in Abhängigkeit von Kontext + Form $+\log ($ Dauer $)+(1 / V P)+(0+\log ($ Dauer $) / V P)$, mit erklärter Varianz $R^{2}$.

\begin{tabular}{lcc}
\hline & F1 & F2 \\
\hline (Intercept) & $405,2(48,6)^{* * *}$ & $1822,4(95,0)^{* * *}$ \\
Kontextat FP ps & $64,1(25,1)^{*}$ & $109,0(51,3)^{*}$ \\
Kontextah FP ps & $33,6(13,2)^{*}$ & $46,8(25,8)$ \\
Kontextap FP ps & $-12,1(17,5)$ & $29,7(33,9)$ \\
Kontextas FP pp & $13,1(16,6)$ & $-18,2(32,1)$ \\
Kontextas FP ph & $-2,5(13,0)$ & $-66,5(24,9)^{* *}$ \\
Kontextah FP pp & $30,3(20,8)$ & $61,4(41,7)$ \\
FormVN & $50,3(11,3)^{* * *}$ & $-44,0(21,4)^{*}$ \\
log(Dauer) & $33,8(11,4)^{* *}$ & $-36,4(19,0)$ \\
\hline AIC & 3450,9 & 3858,8 \\
Num. obs. & 303 & 305 \\
Num. groups: vp & 8 & 8 \\
\hline $\mathrm{R}_{m}^{2} / \mathrm{R}_{c}^{2}$ & $0,07 / 0,62$ & $0,08 / 0,3$ \\
\hline${ }^{* * *} p<0,001,{ }^{* *} p<0,01,{ }^{*} p<0,05$ &
\end{tabular}

Tabelle 5.19: Signifikante Kontraste für multiple paarweise Vergleiche der positionalen Faktorausprägungen, mit Schätzer $\beta$ und Standardabweichung (s) für $\mathrm{F}_{2}$, Freiheitsgraden (df), 95\%-Konfidenzintervall (KI) und t-Statistik, korrigiert für multiples Testen nach Tukey (Turnpause-FP-Segment $=$ at FP ps, Atmung-FP-Segment $=a h$ FP ps, Segment-FPAtmung $=$ as FP ph).

\begin{tabular}{lrrrrrrr}
\hline Vergleich & $\beta$ & $\mathrm{s}$ & $\mathrm{df}$ & Unteres KI & Oberes KI & $\mathrm{t}$ & $\mathrm{p}$ \\
\hline at FP ps - as FP ph & 175,5 & 52,8 & 289,7 & 18,79 & 332,18 & 3,33 & $<0,05$ \\
ah FP ps - as FP ph & 113,3 & 30,2 & 293,2 & 23,59 & 202,94 & 3,75 & $<0,01$ \\
\hline
\end{tabular}

Ähnlich wie bei den Ergebnissen für die Grundfrequenz wirken sich also Kontexte, die potenziell prosodische Phrasen beginnen können (linkerhand eine Turnpause oder Atmung, rechterhand ein Laut), signifikant auf die Lage und Höhe der Vokale in Füllpartikeln aus. Der Kontext erklärt somit einen Teil der Formvariation von Füllpartikeln.

Vokale in V-Formen liegen im Allgmeinen signifikant höher und anteriorer als VNFormen. Die Dauer hat einen signifikant positiven Einfluss auf $\mathrm{F}_{1}$ (je länger, desto tiefer) und einen signifikant negativen Einfluss auf $\mathrm{F}_{2}$ (je länger, desto posteriorer).

Für diese Unterschiede der Vokalqualitäten je Form gibt es keine zufriedenstellende Erklärung. Da die Messung in der Vokalmitte erfolgte, sollte der Einfluss des nachfolgenden Nasals gering sein - zudem wirken sich zumindest im Englischen Na- 
sale nach Vokalen nicht auf den ersten Formanten des Vokals aus (vgl. Styler 2017: 2476). Möglich ist, dass aufgrund der generell kürzen Vokaldauer in VN-Formen (vgl. Abbildung 5.5 und Tabelle 5.6) die Vokale in VN-Formen nicht ihr eigentliches Target erreichen können (wenn man davon ausgeht, dass die Vokale in beiden Formen dasselbe zugrundeliegende Target haben). Dies spiegelt sich in der Abhängigkeit von $\mathrm{F}_{1}$ und $\mathrm{F}_{2}$ von der Dauer. Neben diesen Erkenntnissen ist besonders die Erklärungskraft der Kontexte für die Vokalvariabilität aufschlussreich.

\subsubsection{Phonationsart}

Für die direkten sequenziellen Kontexte mit der absoluten Häufigkeit von $n>5$ zeigt Abbildung 5.27 die absoluten und relativen Werte je Phonationsart. Glottalisierte Vokale stehen nur in solchen Kontexten, in denen im Ante- oder Postzedens ein Segment oder Click vorkommt (außer in at FP ps), nicht jedoch in interpausaler Position ( $a h$ FP pp, ap FP pp, ah FP ph). Somit ist die Vokalphonation nicht unabhängig von den sequenziellen Kontexte (beidseitiger exakter Fisher-Test basierend auf 2000 Simulationen, $p<0,01)$.

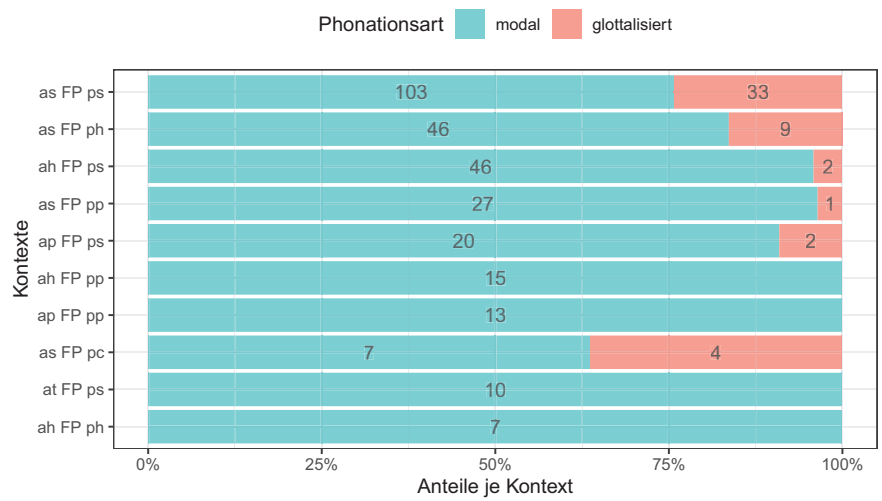

Abbildung 5.27: Absolute und relative Werte der Phonationsart in adjazenten sequenziellen Kontexten $(n>5)$ in GECO-FP (FP = nicht-glottale Füllpartikel, Antezedentia: at = Turn, as $=$ Segment, ap = Pause, ah = Atmungspause; Postzedentia: $\mathrm{ps}=$ Segment, $\mathrm{pp}=$ Pause, $\mathrm{ph}=$ Atmungspause, $\mathrm{pc}=$ Click). 


\subsubsection{Dialogzüge}

Tabelle 5.20 zeigt die Häufigkeiten der Annotationswerte je Dialogzugkategorie, die über die Schwelle von 25 Vorkommen hinauskommen. ${ }^{120}$ Die Anzahl der verschiedenen Dialogzüge und die Anzahl der Wörter je Dialogzug hängt stark von den Annotationsrichtlinien ab. Zur Vergleichbarkeit mit anderen Studien gebe ich daher die prozentuale Verteilung der Wörter über die Dialogzüge an. Die in den Dialogzügen enthaltenen Anteile glottaler (FG) und nicht-glottaler Füllpartikeln (FV) unterscheiden sich signifikant von den erwarteten Häufigkeiten ${ }^{121}\left(\chi^{2}=22,6\right.$, simulierter p-Wert basierend auf 2000 Wiederholungen: $p<0,05)$. Nach Post-hocVergleichen (Fisher-Test nach Shan \& Gerstenberger (2017)) sind dafür KoMPLEXE Antworten, ErzäHLUngen und Trunkierte ERzÄHLUnGEn verantwortlich. In Komplexen Antworten treten glottale Füllpartikeln signifikant seltener auf, als erwartet (4 mal statt 10 mal, $p<0,05$ ). Auch in ERZÄHLUnGEN treten glottale Füllpartikeln signifikant seltener auf, als erwartet (83 mal statt 71 mal, $p<0,01$ ). In TRUnkiERTEN ERzÄHLUNGEN jedoch treten sie überzufällig häufig auf (6 mal statt 3 mal, $p<0,05)$.

Die relative Häufigkeit nicht-glottaler Füllpartikeln über die Wörter in einer Dialogzugkategorie (Spalte FV/W) ist für KOMPLEXe Antworten (3,1\%), unsichere Antworten (2,9\%) und negative Antworten (2,6\%) am höchsten. Die Anzahl der Wörter und die Anzahl der nicht-glottalen Füllpartikeln sind nicht unabhängig voneinander $\left(\chi^{2}=144,1, p<0,001\right)$. Negative Antworten enthalten im Vergleich zur ihren enthaltenen Wörtern signifikant häufiger nicht-glottale Füllpartikeln, als erwartet (17 mal statt 7 mal, $p<0,001)$; ebenso verhält es sich für UNKLARE ANTWORTEN (28 mal statt $10 \mathrm{mal}, p<0$,001) und für KOMPLEXE ANTWORTEN (48 mal statt 16 mal, $p<0,001$ ). BACKCHANNELZÜGE (4 mal statt 28 mal, $p<0,001)$ und ERzÄHLUNGEN (284 mal statt 317 mal, $p<0,001$ ) enthalten signifikant weniger FV-Formen als erwartet.

Dasselbe Vorgehen bestätigt zwar auch einen signifikanten Unterschied zwischen der beobachteten und erwarteten Häufigkeit von Wörtern und glottalen Formen $\left(\chi^{2}=23,7, d f=, p<0,05\right)$, allerdings geht aus den Post-hoc-Tests hervor, dass dies

\footnotetext{
${ }^{120}$ Die Schwelle wurde so gewählt, dass die Summe der ausgeschlossenen Werte ca. $1 \%$ aller in GECO-FP annotierten Werte beträgt. Ausgeschlossen wurden av-n (11), aw-u (10), fe-t (3), fw-t (3), i (3), ae-t (2), av-u (1), aw-fe (1), aw-t (1), und v (1).

${ }^{121}$ Ohne die Zeile mit dem Dialogzug $r$ (Redebereitschaft).
} 
Tabelle 5.20: Häufigkeit der Annotationswerte (n), Wörter (W), Anteil der Wörter (W/n), Anteil der Wörter je Dialogzug am Datensample, Anzahl glottaler (FG) und nicht-glottaler (FV) Füllpartikeln, relative Häufigkeit nicht-glottaler Füllpartikeln $(\mathrm{FV} / \mathrm{W})$, sowie für FV die Anzahl der vokalischen (V), vokalisch-nasalen (VN) und nasalen (N) Formen und ihre Summe je Dialogzug $(\mathrm{n}>25)$ in GECO-FP (ae-p/ae-n/ae-u/aw = Pos./NEG./UNKLARE/ Komplexe Antwort, av-p = Pos. Antwort auf Vergewisserungsfrage, $b=$ Back-

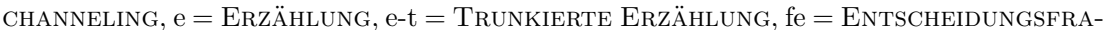
$\mathrm{Ge}, \mathrm{fv}=$ Vergewisserungsfrage, $\mathrm{fw}=$ W-Frage, $\mathrm{r}=$ Redebereitschaft), sortiert nach $\mathrm{FV} / \mathrm{W}$.

\begin{tabular}{l|rrrr|rrr|rrrr}
\hline DZ & $\mathrm{n}$ & Wörter $(\mathrm{W})$ & $\mathrm{W} / \mathrm{n}$ & $\mathrm{W} / \Sigma(\mathrm{W})$ & $\mathrm{FG}$ & $\mathrm{FV}$ & $\mathrm{FV} / \mathrm{W}$ & $\mathrm{V}$ & $\mathrm{VN}$ & $\mathrm{N}$ & $\Sigma$ \\
\hline aw & 105 & 1542 & 14,7 & $3,5 \%$ & 4 & 48 & $3,1 \%$ & 14 & 31 & 2 & 47 \\
ae-u & 28 & 973 & 34,8 & $2,2 \%$ & 3 & 28 & $2,9 \%$ & 5 & 16 & 4 & 25 \\
ae-n & 53 & 666 & 12,6 & $1,5 \%$ & 1 & 17 & $2,6 \%$ & 8 & 6 & 2 & 16 \\
fe & 220 & 1718 & 7,8 & $3,9 \%$ & 1 & 21 & $1,2 \%$ & 10 & 10 & 0 & 20 \\
e-t & 44 & 738 & 16,8 & $1,7 \%$ & 6 & 8 & $1,1 \%$ & 4 & 3 & 0 & 7 \\
ae-p & 123 & 1764 & 14,3 & $4,1 \%$ & 6 & 18 & $1 \%$ & 11 & 6 & 1 & 18 \\
e & 835 & 31604 & 37,8 & $72,6 \%$ & 83 & 284 & $0,9 \%$ & 115 & 144 & 9 & 268 \\
fw & 143 & 793 & 5,5 & $1,8 \%$ & 0 & 6 & $0,8 \%$ & 3 & 2 & 1 & 6 \\
av-p & 41 & 449 & 11 & $1 \%$ & 1 & 2 & $0,4 \%$ & 1 & 1 & 0 & 2 \\
fv & 70 & 442 & 6,3 & $1 \%$ & 0 & 1 & $0,2 \%$ & 0 & 1 & 0 & 1 \\
b & 1863 & 2791 & 1,5 & $6,4 \%$ & 0 & 4 & $0,1 \%$ & 3 & 0 & 0 & 3 \\
r & 47 & 66 & 1,4 & $0,2 \%$ & 0 & 0 & $0 \%$ & 0 & 0 & 0 & 0 \\
\hline$\Sigma$ & 3572 & 43546 & & $100 \%$ & 105 & 437 & & 174 & 220 & 19 & 413 \\
\hline
\end{tabular}

hauptsächlich auf die signifikant höhere FG-Häufigkeit in der Kategorie ABGEBROChENE ERzÄHLUngen ( 6 mal statt 2 mal, $p<0,01$ ) und die signifikant niedrigere Häufigkeit in der Kategorie BACKCHANNELING zurückzuführen ist (0 mal statt 7 mal, $p<0,01)$. Kritisch anzumerken ist, ob im Fall der ABGEBrochenen ERzÄHLung nicht eher Abbrüche denn Füllpartikeln annotiert sind, da Abbrüche im Deutschen mit glottalem Plosiv markiert werden können (Schwitalla 2012). Diese Kategorisierung ist jedoch auch eine Frage der funktionalen Perspektive und wird in dieser Arbeit ausgeblendet.

Die nicht-glottalen Füllpartikeln in Tabelle 5.20 sind nochmals detaillierter für ihre drei häufigsten abstrahierten Formen V, VN und N genauer aufgelistet. ${ }^{122}$ Ihre beobachteten Häufigkeiten unterscheiden sich jedoch nicht signifikant von den erwarteten Häufigkeiten, d. h. ihre Verteilung scheint unabhängig von den Dialogzü-

\footnotetext{
${ }^{122}$ Die Summe für V, VN und N differiert von FV aufgrund von anderen Formen wie beispielsweise Sequenzen von Vokalen und Konsonanten, die hier jedoch aufgrund ihrer kleinen Zahl nicht berücksichtigt werden.
} 
gen zu sein $\left(\chi^{2}\right.$-Text mit simuliertem p-Wert, basierend auf 2000 Wiederholungen; $\left.\chi^{2}=17,78, p=0,53\right)$.

Welche Positionen nehmen Füllpartikeln innerhalb der Dialogzüge ein? Für V und VN-Füllpartikeln sind die Dialogzüge mit $n>7$ Vorkommen in Tabelle 5.21 aufgeführt. Füllpartikelpositionen können in der EMU-Datenbank für Anfang, Mitte und Ende eines Dialogzuges ermittelt werden, wobei ,Anfang' als Gleichheit der linken Intervallgrenze von Dialogzug und Füllpartikel und ,Ende‘ als Gleichheit der rechten Intervallgrenze definiert ist. ,Mitte ${ }^{6}$ bezeichnet alle sonstigen Positionen. V- und VN-Formen sind für sich genommen über die drei Positionen signifikant unterschiedlich verteilt (für V: $\chi^{2}=29,87$, simulierter p-Wert basierend auf 2000 Wiederholungen: $p<0$, 01; für VN: $\chi^{2}=55,53$, simulierter p-Wert basierend auf 2000 Wiederholungen: $p<0,001)$.

Tabelle 5.21: Häufigkeit vokalischer und vokalisch-nasaler Füllpartikeln $(\Sigma(V+V N)>7)$ nach Position im Dialogzug (DZ) und relativem Anteil am absoluten Vorkommen eines DZ in GECO-FP (ae-n/ae-p/ae-u/aw = NEG./POs./UnKLARE/KOMPLEXE AnTwORT, e = ERZÄHLUnG, fe = ENTSCHEIDUNGSFRAGE).

\begin{tabular}{|c|c|c|c|c|c|c|c|c|c|c|c|c|c|}
\hline \multirow{2}{*}{$\frac{\mathrm{DZ}}{\mathrm{ae}-\mathrm{n}}$} & \multicolumn{2}{|c|}{ Anfang (\%) } & \multicolumn{2}{|c|}{$\begin{array}{c}\text { äh } \\
\text { Mitte (\%) }\end{array}$} & \multicolumn{2}{|c|}{ Ende (\%) } & \multicolumn{2}{|c|}{ Anfang (\%) } & \multicolumn{2}{|c|}{$\begin{array}{c}\text { ähm } \\
\text { Mitte (\%) }\end{array}$} & \multicolumn{2}{|c|}{ Ende $(\%)$} & \multirow{2}{*}{$\begin{array}{c}\Sigma \\
14\end{array}$} \\
\hline & 2 & $(3,8)$ & 6 & $(11,3)$ & 0 & $(0)$ & 2 & $(3,8)$ & 4 & $(7,5)$ & 0 & $(0)$ & \\
\hline ae-p & 3 & $(2,4)$ & 8 & $(6,5)$ & 0 & (0) & 1 & $(0,8)$ & 5 & $(4,1)$ & 0 & (0) & 17 \\
\hline ae-u & 1 & $(3,6)$ & 4 & $(14,3)$ & 1 & $(3,6)$ & 5 & $(17,9)$ & 11 & $(39,3)$ & 1 & $(3,6)$ & 23 \\
\hline aw & 5 & $(4,8)$ & 10 & $(9,5)$ & 0 & (0) & 13 & $(12,4)$ & 18 & $(17,1)$ & 1 & (1) & 47 \\
\hline $\mathrm{e}$ & 3 & $(0,4)$ & 114 & $(13,7)$ & 5 & $(0,6)$ & 3 & $(0,4)$ & 140 & $(16,8)$ & 2 & $(0,2)$ & 267 \\
\hline fe & 2 & $(0,9)$ & 7 & $(3,2)$ & 1 & $(0,5)$ & 0 & $(0)$ & 9 & $(4,1)$ & 1 & $(0,5)$ & 20 \\
\hline$\Sigma$ & 16 & & 149 & & 7 & & 24 & & 187 & & 5 & & 388 \\
\hline
\end{tabular}

Nach Post-hoc-Tests für alle drei Positionen und sechs Dialogzüge sind V-Formen am Anfang KOMPLEXER ANTwORTEN signifikant häufiger als erwartet (5 mal statt 1 mal, $p<0,01)$, in ihrer Mitte jedoch seltener (10 mal statt 13 mal, $p<0,05)$. In ERZÄHLUNGEN sind V-Formen anfangs signifikant seltener (3 mal statt 11 mal, $p<0,001)$ und mittig signifikant häufiger als erwartet (114 mal statt 106 mal, $p<$ $0,001)$.

VN-Formen zeigen für Erzählungen das gleiche Verhalten. In Antworten auf WFragen sind auch sie zu Beginn signifikant häufiger als erwartet (13 mal statt 4 mal, $p<0,001$ ); zudem sind sie gleichzeitig mittig signifikant seltener als erwartet (18 mal statt 28 mal, $p<0,001)$. Wie auch die V-Formen sind die VN-Formen in ERzÄH- 
LUNGEN anfangs signifikant seltener (3 mal statt 16 mal, $p<0,001$ ) und mittig signifikant häufiger als erwartet (140 mal statt $126 \mathrm{mal}, p<0,001)$. VN-Formen sind zudem in UNKLAREN ANTWORTEN anfangs signifikant häufiger (5 mal statt 2 mal, $p<0,05$ ) und mittig signifikant seltener als erwartet (11 mal statt 15 mal, $p<0,05$ ).

Vergleicht man V- und VN-Formen jeweils bezüglich ihrer Vorkommen für jede Position einzeln miteinander, finden sich keine signifikanten Unterschiede ( $\mathrm{V}$ vs. VN am Anfang: $\chi^{2}=7,94$, simulierter p-Wert basierend auf 2000 Wiederholungen: $p=0,18$; V vs. VN in der Mitte: $\chi^{2}=5,33$, simulierter $\mathrm{p}$-Wert basierend auf 2000 Wiederholungen: $p=0,4$; V vs. VN am Ende: Exakter Test nach Fisher, $p=0,83$ ). Offen bleibt, inwiefern der Beginn eines Dialogzuges beziehungsweise seine ,Mitte6 konfundierende Variablen wie beispielsweise Intonationsphrasen enthält, weswegen die Aussagekraft dieser Daten mit Vorsicht zu behandeln ist.

Tabelle 5.22 zeigt die Häufigkeitsverteilung der häufigsten nicht-glottalen Füllpartikelkontexte mit mindestens einem Kontext, der öfter als fünfmal in einem der Dialogzüge vorkommt.

Tabelle 5.22: Häufigkeiten nicht-glottaler Kontexte $n \geq 5$, in den Dialogzügen ae-n/ae-p/ae-u/(NEG./POs./unklare/KOMPleXe AntworT), e (ERZÄhlung) und fe (Entscheidungsfrage). Ante- und Postzedentia in den Kontexten beinhalten Atmung (ah/ph), Pausen (ap/pp), Segmente (as/ps) und Clicks (ps). Pfeile kennzeichnen Zeilen, die in die gemischten Modelle für Dauer, $\mathrm{f}_{0}$ und Formanten eingehen.



Je Dialogzug können die Kontexte recht unterschiedlich verteilt sein; so kommt beispielsweise der Kontext Segment-FP-Pause nur in Erzählungen vor, jedoch nicht in Antworten auf W-Fragen. Problematisch sind die geringen oder fehlenden Werte in den einzelnen Zellen besonders für die Auswertung kontinuierlicher Parameter wie 
Dauer, Grundfrequenz und Formanten. Daher betrachte ich für die weitere Auswertung nur die mit Pfeilen markierten Kontexte und die Dialogzüge aw und $e$.

\subsubsection{Dauer}

Die Auswahl der vier häufigsten adjazenten Kontexte und die Auswahl von Dialogzügen mit mindestens fünf Vorkommen führt zur Analyse der Daten in Tabelle 5.23 in einem linearen gemischten Modell.

Tabelle 5.23: Häufigkeit vokalischer und vokalisch-nasaler Füllpartikeln für Dialogzüge in Erzählungen (e) und Komplexen Antworten (aw) in den vier ausgewählten Kontexten (vgl. Tabelle 5.22) in GECO-FP.

\begin{tabular}{c|cc}
\hline & $\mathrm{V}$ & $\mathrm{VN}$ \\
\hline $\mathrm{aw}$ & 10 & 14 \\
$\mathrm{e}$ & 88 & 84 \\
\hline
\end{tabular}

Füllpartikeldauer wird als abhängige Variable, Füllpartikelform, Kontext und Dialogzug als unabhängige Variable modelliert. Versuchspersonen gehen als zufällige Intercepts in das Modell ein. Interaktionen und Random Slopes verbessern das Modell nicht. Abbildung 5.28 und Tabelle 5.24 zeigen die Effekte des Modells.

$1 \mid \operatorname{lmer}($ FP-Dauer $\sim$ Form + Kontext + Dialogzug $+(1 \mid \mathrm{VP})$, data $=\ldots)$

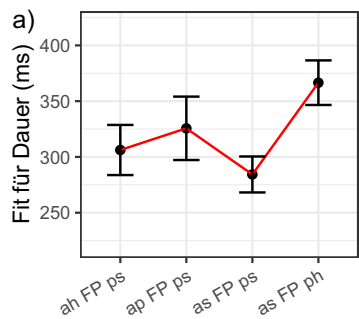

Kontexte b)

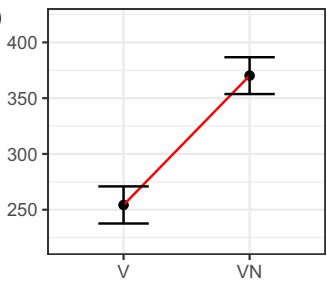

Form c)

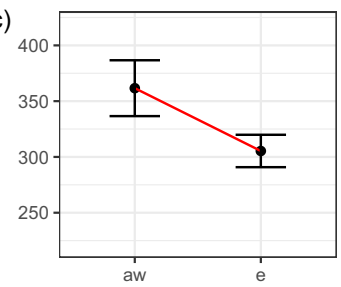

Dialogzüge

Abbildung 5.28: Modelleffekte für Füllpartikeldauern in a) sequenziellen Kontexten für Atmung-FP-Segment- (ah FP ps), Pause-FP-Segment- (ap FP ps), Segment-FP-Segment(as FP ps) und Segment-FP-Atmung-Kontexte (as FP ph), b) für Form und c) für Dialogstruktur (aw = KOMPLEXe ANTwORT, e = ERZÄHLUNG) in GECO-FP.

Wie in Abschnitt 5.2.1.1 sind vokalisch-nasale Formen signifikant länger als vokalische $(\beta=115,9 \mathrm{~ms})$, während Füllpartikeln in Kontexten, in denen die Füll- 
partikel zwischen einem Segment und Atmungspause steht, ca. $60 \mathrm{~ms}$ länger sind als Füllpartikeln in dem umgekehrten Kontext Atmung-FP-Segment (aufgrund der Verlangsamung nach Segmenten und vor Atmung wieder ein Indiz für prosodische Phrasen, vgl.Abschnitt 6.2.3.1). Der Kontext Segment-FP-Segment ist zudem post hoc signifikant kürzer als der Kontext Segment-FP-Atmung (Vergleich nach Tukey, Werte für as FP ps - as FP $p h, \beta=-82,3, s=19,9, d f=188,2, K I_{95 \%}=$ $[-133,7,-30,8], t=-4,1, p<0,001)$. Die Füllpartikellänge in ErzäHLUNGEN ist signifikant kürzer $(-56 \mathrm{~ms})$ als in KOMPLEXEN ANTwORTEN (Abbildung 5.28 c). Dies könnte ein Indiz für eine erhöhte Planung bei Antworten sein.

Tabelle 5.24: Lineares gemischtes Modell für die Füllpartikeldauer in Abhängigkeit von Dialogzug + Kontext + Form $+(1 / V P)$, mit erklärter Varianz $R^{2}$.

\begin{tabular}{lc}
\hline & Dauer \\
\hline (Intercept) & $297,7(29,7)^{* * *}$ \\
FormVN & $115,9(17,4)^{* * *}$ \\
Kontextap FP ps & $19,4(31,3)$ \\
Kontextas FP ps & $-21,9(22,3)$ \\
Kontextas FP ph & $60,3(25,6)^{*}$ \\
Dialogzuge & $-56,3(24,1)^{*}$ \\
\hline AIC & 2350,0 \\
Num. obs. & 196 \\
Num. groups: vp & 8 \\
\hline $\mathrm{R}_{m}^{2} / \mathrm{R}_{c}^{2}$ & $0,32 / 0,38$ \\
\hline${ }^{* * *} p<0,001,{ }^{* *} p<0,01,{ }^{*} p<0,05$
\end{tabular}

\subsubsection{Grundfrequenz}

Da aus Abschnitt 5.2.1.2 hervorgeht, dass sich V- und VN-Formen bezüglich der Grundfrequenz nicht signifikant unterscheiden und ein Modellvergleich auch keinen Unterschied belegt, werden diese Formen nicht separat im Modell betrachtet. Das beste Modell für die Grundfrequenz beinhaltet Kontext, Dialogzüge, und ihre Interaktion als unabhängige Variable. Versuchspersonen gehen als zufällige Intercepts in das Modell ein. Random Slopes verbessern das Modell nicht.

$1 \mid \operatorname{lmer}(\mathrm{FP}-$ Dauer $\sim$ Kontext $*$ Dialogzug $+(1 \mid \mathrm{VP})$, data $=\ldots)$

Abbildung 5.29 zeigt den Interaktionseffekt von Kontexten und Dialogzügen auf die Grundfrequenz, Tabelle 5.25 ihre Werte. In KOMPLEXen AnTwOrTEN werden Füll- 
partikeln in den Kontexten Pause-FP-Segment (ap FP ps) und Segment-FP-Atmung (as FP ph) signifikant tiefer geäußert als im Kontext Atmung-FP-Segment (ah FP $p s)$. Dies deutet zumindest für Atmung-FP-Segment vs. Segment-FP-Atmung wieder auf einen Effekt der Position zu Beginn beziehungsweise zum Ende einer Intonationsphrase hin.
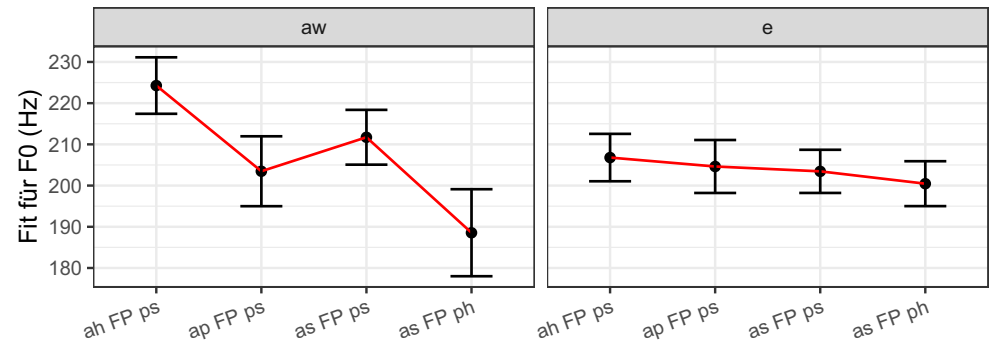

Sequenzielle Kontexte

Abbildung 5.29: V- und VN-Füllpartikel- $f_{0}$ für sequenzielle Kontexte Atmung-FPSegment- (ah FP ps), Pause-FP-Segment- (ap FP ps), Segment-FP-Segment- (as FP ps) und Segment-FP-Atmung (as FP ph) und Dialogstruktur (aw = Komplexe AnTwort, e $=$ ERZÄHLUNG GECO-FP.

Tabelle 5.25: Linear gemischte Modelle für die Füllpartikeldauer in Abhängigkeit von Dialogzug + Kontext $+(1 / V P)$, mit erklärter Varianz $R^{2}$.

\begin{tabular}{lc}
\hline & Grundfrequenz \\
\hline (Intercept) & $224,3(6,9)^{* * *}$ \\
Kontextap FP ps & $-20,8(8,3)^{*}$ \\
Kontextas FP ps & $-12,5(6,5)$ \\
Kontextas FP ph & $-35,7(10,5)^{* * *}$ \\
Dialogzuge & $-17,5(5,5)^{* *}$ \\
Kontextap FP ps:Dialogzuge & $18,6(9,6)$ \\
Kontextas FP ps:Dialogzuge & $9,2(7,2)$ \\
Kontextas FP ph:Dialogzuge & $29,4(11,0)^{* *}$ \\
\hline AIC & 1436,7 \\
Num. obs. & 181 \\
Num. groups: vp & 8 \\
\hline $\mathrm{R}_{m}^{2} / \mathrm{R}_{c}^{2}$ & $0,07 / 0,58$ \\
\hline${ }^{* * *} p<0,001,{ }^{* *} p<0,01,{ }^{*} p<0,05$ &
\end{tabular}

Tabelle 5.26 zeigt die signifikanten Kontraste aus Post-hoc-Tests. Füllpartikeln 
im Kontext Atmung-FP-Segment zeigen eine signifikante höhere $\mathrm{f}_{0}$ in KOMPLEXEN Antworten als in allen vier Kontexten des Dialogzugs ErzäHLung. Offen bleibt, woher dieser Unterschied rührt und ob er in ungesehenen Daten replizierbar ist (da das Signifikanzniveau von $\alpha=0,05$ nur knapp unterschritten wird).

Tabelle 5.26: Signifikante Kontraste für multiple paarweise Vergleiche der positionalen Faktorausprägungen, mit Schätzer $\beta$ und Standardabweichung (s) für $\mathrm{f}_{0}$, Freiheitsgraden (df), $95 \%$-Konfidenzintervall (KI) und t-Statistik, korrigiert für multiples Testen nach Tukey (Atmung-FP-Segment $=$ ah FP ps, Pause-FP-Segment $=a p$ FP $p s$, Segment-FP-Segment $=$ as FP ps, Segment-FP-Atmung $=$ as FP ph, KOMPlexe ANTWOrT $=a w$, ErzählunG $=e$ ) in GECO-FP.

\begin{tabular}{lrrrrrrr}
\hline Vergleich & $\beta$ & $\mathrm{s}$ & $\mathrm{df}$ & Unteres KI & Oberes KI & $\mathrm{t}$ & $\mathrm{p}$ \\
\hline ah FP ps,aw - as FP ph,aw & 35,7 & 10,5 & 167,7 & 3,34 & 68,10 & 3,39 & $<0,05$ \\
ah FP ps,aw - ah FP ps,e & 17,5 & 5,5 & 167,6 & 0,53 & 34,42 & 3,17 & $<0,05$ \\
ah FP ps,aw - ap FP ps,e & 19,6 & 6,3 & 168,6 & 0,19 & 39,10 & 3,10 & $<0,05$ \\
ah FP ps,aw - as FP ps,e & 20,8 & 5,1 & 169,1 & 5,29 & 36,36 & 4,11 & $<0,01$ \\
ah FP ps,aw - as FP ph,e & 23,8 & 5,2 & 168,8 & 7,95 & 39,68 & 4,61 & $<0,001$ \\
\hline
\end{tabular}

\subsubsection{Formanten}

Wirkt sich die Dialogstruktur auf die Vokalqualität einer Füllpartikeln aus? In Tabelle 5.22 sind die Kontexte, die für die kontinuierlichen abhängigen phonetischen Parameter genauer analysiert werden, mit Pfeilen markiert, nämlich ah FP ps, ap FP ps, as FP ps und as FP ph. Im Folgenden vergleiche ich das Modell aus Tabelle 5.22 mit einem neuen Modell, das zusätzlich den Prädiktor Dialogzug enthält, für alle Faktorstufen mit $n>6$ : Positive, NeGative, UnkLARE Und KOMPLEXe ANTworTEN sowie ERzÄHLUnGen und Entscheidungsfragen. ${ }^{123}$ Abhängige Variable ist $\mathrm{F}_{1}$ (in der Mitte des Vokals gemessen). Folgender Beispielcode veranschaulicht das neue Modell in $\mathrm{R}$.

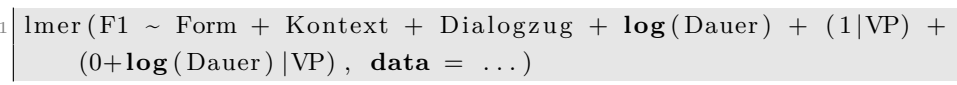

Der zusätzliche Prädiktor Dialogzug verbessert das alte Modell aus Abschnitt 5.2.1.3 in Likelihood-Vergleichen jedoch nicht signifikant $(p=0,8)$. Auch für $\mathrm{F}_{2}$ verbessert sich das Modell nicht $(p=0,1)$. Für $\mathrm{F}_{3}$ wird ein Modell mit Dialogzügen gegen

\footnotetext{
${ }^{123}$ Hierzu müssen allerdings alle sieben sequenziellen Kontexte aus Tabelle 5.22 berücksichtigt werden, also auch as FP pp, at FP ps und ah FP pp.
} 
ein Nullmodell verglichen. Das Nullmodell ist signifikant besser $(p<0,05)$. Somit besteht keine Evidenz für einen Einfluss der Dialogstruktur auf die Vokalqualität.

Aus Tabelle 5.20 geht hervor, dass ERzÄHLungen und KOMPLEXe AnTwOrTen absolut die größte Anzahl an Füllpartikeln zeigen. Zusätzlich zeigt Tabelle 5.22, dass intersegmentale Füllpartikeln absolut in den neun häufigsten Kontexten jeweils am häufigsten sind. Daher rechne ich ein neues Modell mit den reduzierten Daten in Tabelle 5.27, in das als Dialogzugausprägungen nur ERZÄHLUNGEN und KOMPLEXE AnTwORTEN eingehen und Füllpartikeln in intersegmentalen Kontexten mit allen anderen Kontexten in einem binären Faktor bin.Kontext kontrastiert werden, was die Datenabdeckung deutlich erhöht.

Tabelle 5.27: Anzahl der für Formanten analysierten Füllpartikelformen in KOMPLEXEN ANTWORTEN und ERZÄHLUNGEN in intersegmentalen (as FP ps) und nicht-intersegmentalen (anderen) Kontexten in GECO-FP.

\begin{tabular}{ll|rr}
\hline Dialogzug & bin.Kontext & V & VN \\
\hline aw & as FP ps & 3 & 6 \\
aw & andere & 11 & 25 \\
e & as FP ps & 46 & 41 \\
e & andere & 68 & 101 \\
\hline
\end{tabular}

Für $\mathrm{F}_{1}$ ist ein Modell mit den Daten in Tabelle 5.27 signifikant besser als ein Modell, welches nur sequenzielle Kontexte enthält $(p<0,01)$. Für $\mathrm{F}_{2}$ und $\mathrm{F}_{3}$ verbessert der Faktor Dialogzug das Modell wiederum nicht (für $\mathrm{F}_{2} p=0$, 7; für $\mathrm{F}_{1}$ $p=1$ ). Abbildung 5.30 und Tabelle 5.28 zeigen die Anpassungen für das beste Modell für $\mathrm{F}_{1}$ mit Füllpartikelform und logarithmisierter Dauer sowie der Interaktion zwischen Dialogzügen und sequenziellen Kontexten in Abbildung 5.30 a als unabhängigen Variablen, mit Versuchspersonen als Random Intercepts und der logarithmisierten Füllpartikeldauer je Versuchsperson als Random Slopes.

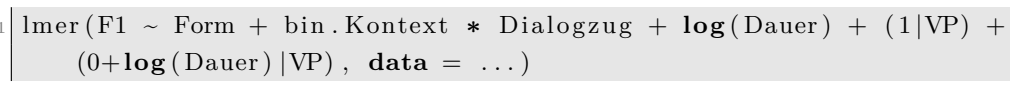

Die Höhe des Füllpartikelvokals liegt in nicht-intersegmentalen Kontexten für KOMPLEXE ANTwORTEN signifikant tiefer im Vokalraum ( $\mathrm{F}_{1}$ ist um $67 \mathrm{~Hz}$ höher) als in nicht-intersegmentalen Kontexten in ERZÄHLUNGEN und auch signifikant tiefer im Vokalraum ( $\mathrm{F}_{1}$ ist $\mathrm{um} 60 \mathrm{~Hz}$ höher) als in intersegmentalen Kontexten in ERzÄHLungen (vgl. die Post-hoc-Tests in Tabelle 5.29). Innerhalb KomPLEXer ANT- 


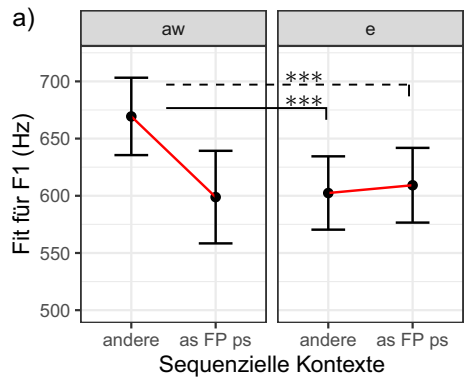

b)



c)

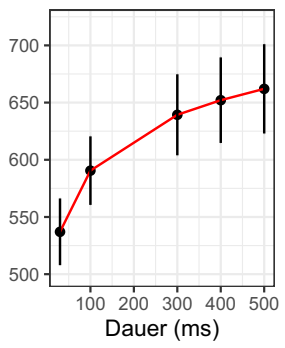

Abbildung 5.30: Modelleffekte für $F_{1}$ in Abhängigkeit von a) sequenziellen Kontexten (intersegmental $=$ as FP ps; nicht-intersegmental $=$ andere $)$ je Dialogzug $($ aw $=$ KOMPLEXE Antwort, e = ERzÄHLUnG), b) Form (V = vokalisch, VN = vokalisch-nasal) und c) Dauer in GECO-FP. Signifikante Kontraste in (a) ergeben sich aus Tabelle $5.29\left({ }^{* * *} p<0,001\right)$.

Tabelle 5.28: Lineares gemischtes Modell für $\mathrm{F}_{1}$ in Abhängigkeit von Form + bin. Kontext * Dialogzug $+\log ($ Dauer $)+(1 / V P)+(0+\log ($ Dauer $) / V P)$, mit erklärter Varianz $R^{2}$.

\begin{tabular}{lc}
\hline & $\mathrm{F}_{1}$ \\
\hline (Intercept) & $334,6(60,1)^{* * *}$ \\
FormVN & $67,2(11,1)^{* * *}$ \\
bin.Kontextandere & $70,5(28,6)^{*}$ \\
Dialogzuge & $10,4(27,1)$ \\
log(Dauer) & $44,4(11,2)^{* * *}$ \\
bin.Kontextandere:Dialogzuge & $-77,3(30,1)^{*}$ \\
\hline AIC & 3174,6 \\
Num. obs. & 279 \\
Num. groups: vp & 8 \\
\hline $\mathrm{R}_{m}^{2} / \mathrm{R}_{c}^{2}$ & $0,11 / 0,64$ \\
\hline${ }^{* * *} p<0,001,{ }^{* *} p<0,01,{ }^{*} p<0,05$ &
\end{tabular}

WORTEN ist der Unterschied zwischen intersegmentalen und nicht-intersegmentalen Kontexten nicht signifikant. Nicht-intersegmentale Kontexte innerhalb von KOMPLEXEN Antworten sind demnach verschieden von allen Kontexten in ERzÄHLUnGEN. Dies würde bedeuten, dass Füllpartikeln in nicht-intersegmentaler Position von KOMPLEXEN ANTWORTEN beeinflusst werden. 
Tabelle 5.29: Signifikante Kontraste für multiple paarweise Vergleiche der Faktorausprägungen für Dialogzüge (aw = KOMPLEXen ANTworT, e = ERzÄHLunG) und sequenziellen Kontexten (intersegmental $=$ as FP ps, nicht-intersegmental $=$ andere), mit Schätzer $\beta$ für $\mathrm{F}_{1}$, Standardabweichung (s), Freiheitsgraden (df), 95\%-Konfidenzintervall (KI) und t-Statistik, korrigiert für multiples Testen nach Tukey.

\begin{tabular}{l|rrrrrrr}
\hline Vergleich & $\beta$ & $\mathrm{s}$ & $\mathrm{df}$ & Unteres KI & Oberes KI & $\mathrm{t}$ & $\mathrm{p}$ \\
\hline andere,aw - as FP ps,e & 60,16 & 15,57 & 267,09 & 19,92 & 100,41 & 3,86 & $<0,001$ \\
andere,aw - andere,e & 66,95 & 14,29 & 267,86 & 30,00 & 103,91 & 4,68 & $<0,001$ \\
\hline
\end{tabular}

Im Folgenden untersuche ich, ob sich die beiden Kontext- und Dialogzuggruppen nicht nur für Mittelpunktmessungen voneinander unterscheiden, sondern ob möglicherweise auch die Formanttrajektorien der Dialogzüge ERzÄHLUnG und KOMPLEXE ANTWORT zwischen intersegmentalen nicht-intersegmentalen Kontexten changieren. Hierzu nutze ich generalisierte additive Modelle (GAM), die die zeitnormalisierten und lobanovnormalisierten $\mathrm{F}_{1}$-Werte in Abhängigkeit von Füllpartikelform, Kontext und Dialogzug in dem Interaktionsprädiktor DZ.Form.Kontext berücksichtigen und mit Likelihood-Tests (ML-gefittet) verglichen werden. Das beste Modell (vgl. RCode unten) enthält die Interaktion aller drei Prädiktoren (Z.1), Kurvenglättungen für diese Prädiktoren (Z.2) sowie zufällige Kurvenglättungen über die Prädiktoren je Versuchsperson (Z.3) und eine Korrektur für Autokorrelation (Z.4).

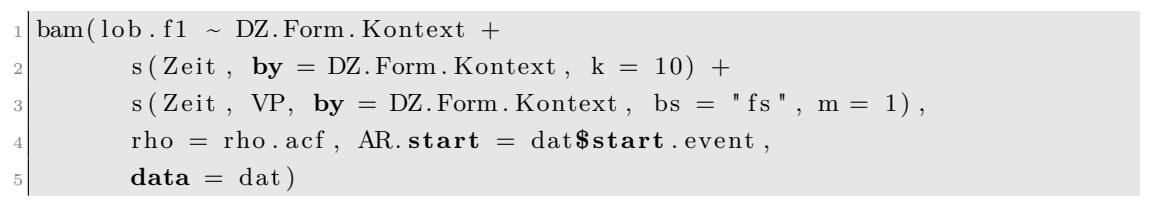

Abbildung 5.31 stellt die im Modell angepassten Trajektorien für $\mathrm{F}_{1}$ und die Differenzen ihrer Kurven dar. Signifikante Unterschiede der Trajektorien sind vorhanden, wenn die Differenzkurve von der Nulllinie abweicht. In Abbildung $5.31 \mathrm{a}-\mathrm{d}$ ist dies nicht der Fall. In $5.31 \mathrm{f}$ weichen die Trajektorien der V-Füllpartikeln in nicht-intersegmentalen Kontexten (5.31 e) zwischen $8 \%$ und $100 \%$ der normalisierten Zeit signifikant voneinander $\mathrm{ab}$; in $5.31 \mathrm{~h}$ ist dies für die $\mathrm{F}_{1}$-Trajektorie der beiden Kurven in $5.31 \mathrm{~g}$ zwischen $21 \%$ und $94 \%$ der normalisierten Zeit der Fall. Tabelle 5.30 zeigt die Werte für die im Modell enthaltenen Glättungsaudrücke. In nicht-intersegmentalen Positionen ist $\mathrm{F}_{1}$ für V- und VN-Füllpartikeln also signifikant niedriger, wenn die Füllpartikeln in KOMPLEXEN ANTwORTEN stehen, als wenn sie 

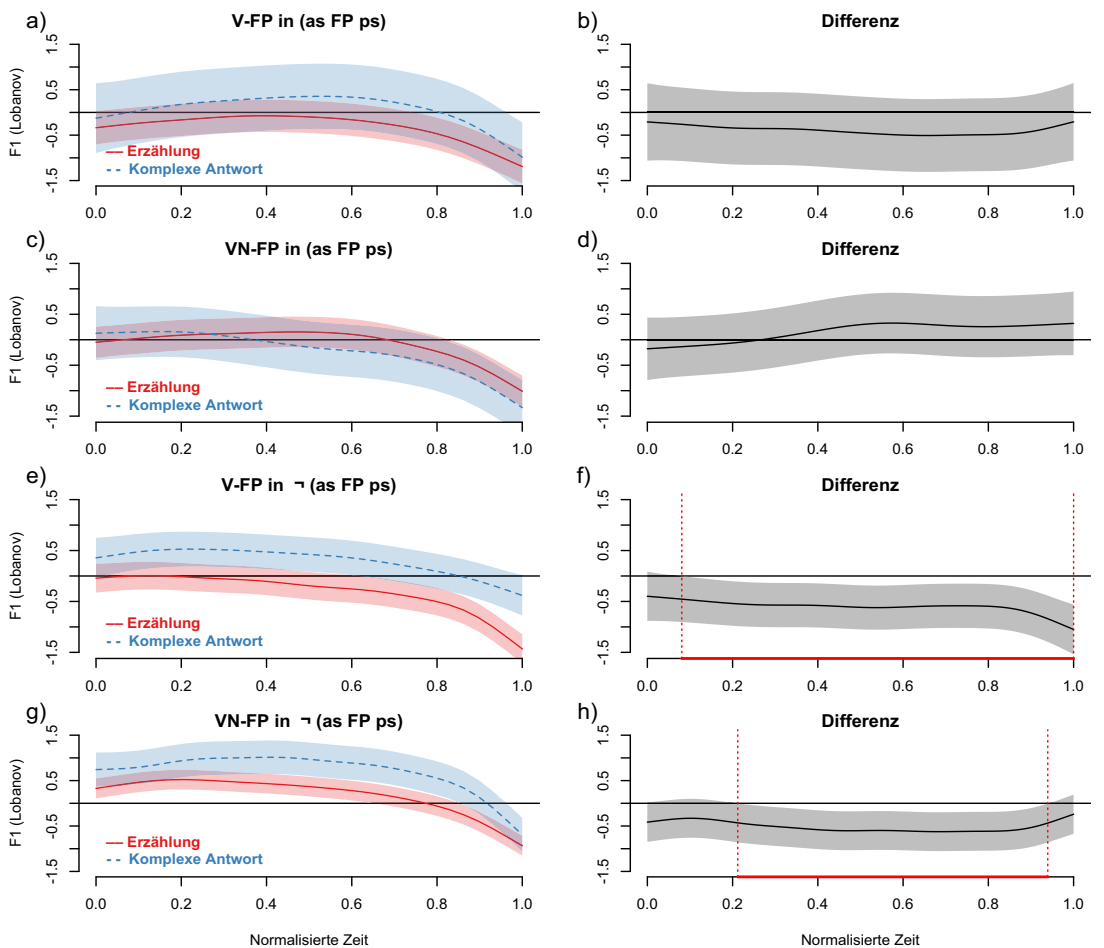

Abbildung 5.31: Angepasste $\mathrm{F}_{1}$-Trajekorien der Vokale in V- und VN-Füllpartikel in intersegmentalen Kontexten (as FP ps) für die Dialogzüge ErzÄHLUng (rot) und KOMPLEXE AnTwORT (blau) in a)-d) in nicht-intersegmentalen Kontexten ( $\neg$ as FP ps) in e)-h) in GECO-FP. Die rechten Bilder zeigen die Differenz der jeweils links abgebildeten Trajektorien; rote Balken kennzeichnen signifikante Abweichungen von der Nulllinie.

in ERZÄHLUngEn vorkommen. Dieser positional bedingte Effekt könnte auf die stillen Pausen und Atmungspausen zurückgehen, die links, rechts, oder beidseits der Füllpartikel auftreten. Zusätzlich könnte hier auch ein prosodisch bedingter Effekt vorliegen, wenn die Füllpartikel am Anfang oder Ende einer Intonationsphrase steht, so dass sie sich in genau diesen nicht-intersegmentalen Kontexten befindet. Daher ist die Einbeziehung der Position innerhalb von Intonationsphrasen für weitere Aussagen über diesen Effekt unbedingt notwendig. 
Tabelle 5.30: Effektive Freiheitsgrade (edf), F- und p-Werte für die unabhängigen (DZ.Form.Kontext) und zufälligen (DZ.Form.Kontext je Versuchsperson) Glättungsausdrücke der Trajektorien für $\mathrm{F}_{1}$ über die normalisierte Zeit.

\begin{tabular}{l|rrr}
\hline & edf & $\mathrm{F}$ & $\mathrm{p}$ \\
\hline s(Zeit):DZ.Form.Kontextaw.V.intersegmental & 4,11 & 3,90 & $<0,01$ \\
s(Zeit):DZ.Form.Kontexte.V.intersegmental & 5,11 & 12,27 & $<0,001$ \\
s(Zeit):DZ.Form.Kontextaw.VN.intersegmental & 4,67 & 7,52 & $<0,001$ \\
s(Zeit):DZ.Form.Kontexte,VN,intersegmental & 6,03 & 18,74 & $<0,001$ \\
s(Zeit):DZ.Form.Kontextaw.V.andere & 3,57 & 3,67 & $<0,01$ \\
s(Zeit):DZ.Form.Kontexte.V.andere & 6,40 & 15,26 & $<0,001$ \\
s(Zeit):DZ.Form.Kontextaw,VN,andere & 7,31 & 31,61 & $<0,001$ \\
s(Zeit):DZ.Form.Kontexte.VN.andere & 6,96 & 37,91 & $<0,001$ \\
s(Zeit,vp):DZ.Form.Kontextaw.V.intersegmental & 1,33 & 0,10 & $<0,05$ \\
s(Zeit,vp):DZ.Form.Kontexte.V.intersegmental & 43,79 & 1,34 & $<0,001$ \\
s(Zeit,vp):DZ.Form.Kontextaw.VN.intersegmental & 7,55 & 0,20 & $<0,05$ \\
s(Zeit,vp):DZ.Form.Kontexte,VN,intersegmental & 30,39 & 0,68 & $<0,001$ \\
s(Zeit,vp):DZ.Form.Kontextaw.V.andere & 8,41 & 0,33 & $<0,01$ \\
s(Zeit,vp):DZ.Form.Kontexte.V.andere & 52,97 & 1,28 & $<0,001$ \\
s(Zeit,vp):DZ.Form.Kontextaw,VN,andere & 15,25 & 0,35 & $<0,001$ \\
s(Zeit,vp):DZ.Form.Kontexte.VN.andere & 47,10 & 1,26 & $<0,001$ \\
\hline
\end{tabular}

Für $\mathrm{F}_{2}$ gibt es wie schon für die Vokalmittelpunkte keine Dialogzugeffekte für $\mathrm{V}$ - und VN-Formen in intersegmentalen und nicht-intersegmentalen Kontexten. ${ }^{124}$ Von der Analyse der $\mathrm{F}_{3}$-Trajektorien wird abgesehen, da ihre Interpretation unklar bleibt.

\subsubsection{Phonationsart}

Für die Verteilung von Füllpartikeln in Dialogzügen mit einer absoluten Häufigkeit von $n>5$ je Dialogzug zeigt Abbildung 5.32 die absoluten und relativen Werte je Phonationsart. Die Verteilung weicht nicht signifikant von den erwarteten Werten ab $(\chi=5,1, d f=7, p=0,65)$. Somit kann der Dialogstruktur kein Effekt bezüglich der Phonationsart zugeschrieben werden.

\footnotetext{
${ }^{124} \mathrm{~F}_{2}$ in nicht-intersegmentalen Kontexten weichen für Erzählungen und komplexe Antworten in dem Zeitfenster von $10 \%-21 \%$ der normalisierten Zeitdauer zwar signifikant voneinander ab, dieser Unterschied ist aber in Tukey-korrigierten Post-hoc-Tests nicht mehr signifikant.
} 


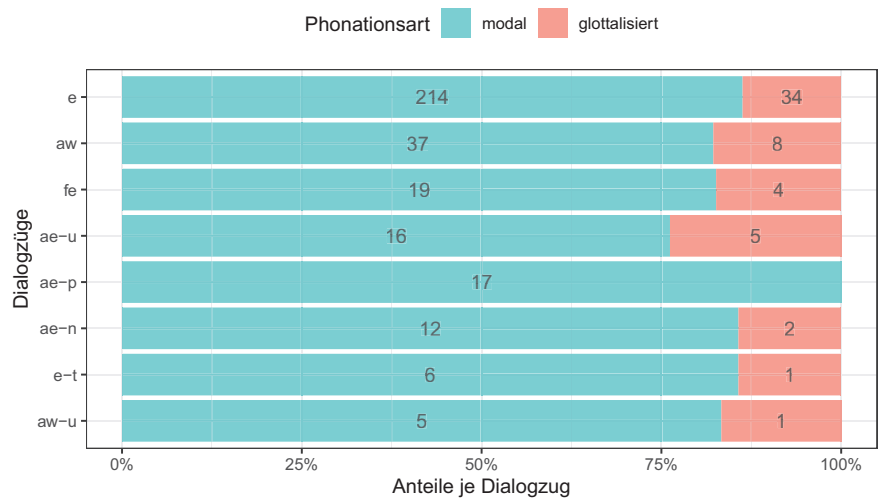

Abbildung 5.32: Absolute und relative Anteile von modalen und glottalisierten Füllpartikeln Dialogzügen $(n>5)$ in GECO-FP (e/e-t = ERzÄHLung/TrunkiERTE ERzÄHLung, ae-p/ae-n/ae-u/aw = Positive/Negative/UnkLare/Komplexe Antwort, fe $=$ EntSCHEIDUNGSFRAGE).

\subsection{Zusammenfassung}

In diesem Teil der Arbeit wurden die in GECO-FP annotierten Füllpartikeln bezüglich ihrer kontextfreien Frequenz, Distribution und phonetischen Form sowie ihre kontextbedingte Abhängigkeit von sequenziellen Kontexten und Dialogzügen explorativ ausgewertet. Im ersten Teil (Abschnitt 5.1) wurde die Hypothese der Formvariabilität untersucht. Die Anzahl der Füllpartikeln in GECO-FP ist nicht mit der Anzahl der Wörter oder Silben je Versuchsperson korreliert, und auch die Sprechgeschwindigkeiten aller Sprecherinnen zeigt keine Korrelation mit der Anzahl ihrer Füllpartikeln je Minute (vgl. Abschnitt 5.1.1). Füllpartikeln zeigen sowohl glottale $(20 \%)$ als auch nicht-glottale Formen ( $80 \%)$, wobei sich die Häufigkeiten beider Gruppen an eine Zipfverteilung annähern (Zipf 1949), d.h. es treten einige häufige und viele seltene Segmentabfolgen auf. Zu den häufigsten Formen nicht-glottaler Füllpartikeln zählen vokalische und vokalisch-nasale Füllpartikeln mit vorangehendem glottalen Plosiv, gefolgt von Formen ohne glottalen Plosiv, gefolgt von GVund GVN-Formen, die vor dem Vokal längere Sequenzen glottaler Plosive zeigen. Orthographisch zusammengefasst sind in GECO-FP ähm-Formen 1,3 mal häufiger als äh-Formen (224 vs. 179). Die häufigsten glottalen Füllpartikeln bestehen aus Se- 
quenzen glottaler Plosive und vereinzelten glottalen Plosiven (vgl. Abschnitt 5.1.2). Die Füllpartikeldauer ist positiv mit der Anzahl ihrer Segmente korreliert. Vokale in VN-Formen sind signifikant kürzer als in V-Formen (vgl. Abschnitt 5.2.1.1). Die Grundfrequenz von nicht-glottalen Füllpartikeln ist interindividuell verschieden und variiert (bei Frauen in der Mitte der Füllpartikel gemessen) zwischen Durchschnittswerten von ca. 187 bis $221 \mathrm{~Hz}$. Dabei liegt die Füllpartikelgrundfrequenz signifikant niedriger als der Durchschnitt aller anderen Äußerungen einer Versuchsperson (vgl. Abschnitt 5.1.4), was jedoch auch auf viele andere Wörter zutreffen wird und zwar Ergebnisse Braun \& Rosin (2015) repliziert, aber nicht sehr aussagekräftig ist. Die akustische Vokalqualität von V-Formen liegt im Bereich von [œ $\bullet$ ə $\varepsilon$ ], die von VNFormen liegt im Bereich von [œ $\bullet$ ə a]. Vier der acht Sprecherinnen unterscheiden die Vokalqualität von V- und VN-Formen signifikant voneinander. Insgesamt liegt der Vokal in VN-Formen signifikant tiefer $(+65 \mathrm{~Hz})$ im Vokalraum als der Vokal in V-Formen. Zwischen zwischen Vokaldauer und Vokalhöhe gibt es eine positive Korrelation im Bereich unter $100 \mathrm{~ms}$ für VN-Formen; in diesem Bereich sind sie noch höher im Vokalraum als über $100 \mathrm{~ms}$. Dies könnte einen target undershoot darstellen, wenn man davon ausgeht, dass das Ziel die ab $100 \mathrm{~ms}$ erreichte Höhe ist (vgl. Abschnitt 4.4.3). Etwa $20 \%$ der V-Füllpartikelvokale werden glottalisiert, aber nur $8,6 \%$ der Vokale in VN-Formen. Modale Vokale in V- und VN-Füllpartikeln sind signifikant länger als glottalisierte Vokale. In den meisten Fällen liegt die Grundfrequenz glottalisierter Vokale unter $140 \mathrm{~Hz}$ oder sie kann nicht gemessen werden (vgl. Abschnitt 5.1.6).

Bis hierhin wurden alle Füllpartikeln ohne nähere Betrachtung ihrer Kontexte betrachtet. Im zweiten Teil der explorativen Auswertung (vgl. Abschnitt 5.2) wird die Form-Kontext-Hypothese anhand zweier ausgewählter Variablen - sequenzielle Kontexte und Dialogstruktur - untersucht. Füllpartikeln zeigen verschiedene Häufigkeiten je Kontext. V-Formen zeigen Präferenzen für Wort-FP-Wort und PauseFP-Wort-Kontexte, während VN-Formen proportional am häufigsten in Wort-FPPause- und Pause-FP-Pause-Kontexten auftreten. Die Wahl einer Füllpartikelform kann nicht auf den sie umgebenden Lautkontext zurückgeführt werden. Füllpartikeln vor oder zwischen Pausen sind signifikant länger als in anderen Kontexten. Dabei ist ihre Dauer unabhängig von der Länge nachfolgender Pausen.

Negative, UnKLARE und KOMPLEXe AnTwORTEN enthalten die höchsten An- 
teile an Füllpartikeln. Nach Segmenten und vor Atmungspausen sind Füllpartikeln mit 63 ms signifikant länger als nach Atmungspausen und vor Segmenten (vgl. Abschnitt 5.2.1.1), was mit einem Positionseffekt einhergehen könnte (finale Längung). In KOMPLEXEN ANTwORTEN sind die Füllpartikeln in diesen Kontexten nochmals deutlich länger $(60+56=116 \mathrm{~ms}$, vgl. Abschnitt 5.2.2.1). Zwar deutet dies prima facie auf einen Planungseffekt hin, kann aber in Berlin Dialogue Corpus compilated v. $1\left(\mathrm{BeDiaCo}_{\mathrm{c}}\right)$ nicht repliziert werden.

Die Grundfrequenz von Füllpartikeln ist zwischen Turnpause oder Atmung und einem nachfolgenden Segment signifikant höher als in intersegmentalen Kontexten. Dies könnte ein Effekt einer neubeginnenden prosodischen Phrase sein. Die Grundfrequenz von nicht-glottalen Füllpartikeln ist nicht nur singulär betrachtet tiefer als die mittlere Grundfrequenz einer Sprecherin (vgl. Abschnitt 5.1.4), sondern auch signifikant tiefer als ihr umgebender segmentaler Kontext (vgl. Abschnitt 5.2.1.2). In KOMPLEXEN ANTwORTEN zeigen Füllpartikeln nach Atmung und vor Segmenten wieder eine signifikant höhere Grundfrequenz als nach Segmenten und vor Atmung, während sich jedoch die Kontexte innerhalb des Dialogzug ERzÄHLUnG nicht voneinander unterscheiden (vgl. Abschnitt 5.2.2.2).

Der Füllpartikelvokal liegt nach Turnpausen oder Atmung und vor Segmenten tiefer im Vokalraum als in intersegmentalen Kontexten - in Turnpause-FP-SegmentKontexten liegt er zudem weiter hinten. Je länger der Vokal ist, desto tiefer und posteriorer wird er artikuliert. Der Vokal liegt in nicht-intersegmentalen Kontexten KOMPLEXER ANTWORTEN signifikant tiefer im Vokaltrakt als in intersegmentalen und nicht-intersegmentalen Kontexten in ERzÄHLUnGEN. Dieser Unterschied gilt für V- und VN-Formen und ist über den größten Teil der $\mathrm{F}_{1}$-Trajektorie robust (vgl. Abschnitt 5.2.2.3), kann aber in $\mathrm{BeDiaCo}_{\mathrm{c}}$ nicht repliziert werden.

Glottalisierte Vokalformen zeigen weder für Kontexte noch für Dialogzüge signifikante Abweichungen von der erwarteten Verteilung je Kontext und Dialogzug (vgl. Abschnitt 5.2.2.4) - sie kommen hauptsächlich intersegmental oder in TurnpauseFP-Segment-Kontexten vor.

Zumindest ein Teil der Formvariabilität von Füllpartikeln ist also tatsächlich kontextbedingt, wobei sequenzielle Kontexte mehr zu Erklärung beitragen als Dialogstruktur. Möglicherweise ist diese zu hierarchisch zu weit entfernt, um sich auf Füllpartikeln auszuwirken. Deutlich wird jedoch für die sequenziellen Mikro- und 
die dialogstrukturellen Makrokontexte, dass die prosodischen Realisierung potenziell konfundierenden beziehungsweise erklärenden Einfluss hat. Daher wird dieser Effekt in der konfirmatorischen Studie auf einer Mesoebene untersucht, indem Intonationsphrasen einbezogen werden. Dieser Teil der konfirmatorischen Studie ist somit wiederum als explorativ anzusehen. Zusätzlich wird nun in der konfirmatorischen Studie überprüft, ob die Ergebnisse der hier vorliegenden explorativen Analyse in ungesehenen Daten repliziert werden können.

Open Access Dieses Kapitel wird unter der Creative Commons Namensnennung 4.0 International Lizenz (http://creativecommons.org/licenses/by/4.0/deed.de) veröffentlicht, wel= che die Nutzung, Vervielfältigung, Bearbeitung, Verbreitung und Wiedergabe in jeglichem Medium und Format erlaubt, sofern Sie den/die ursprünglichen Autor(en) und die Quelle ordnungsgemäß nennen, einen Link zur Creative Commons Lizenz beifügen und angeben, ob Änderungen vorgenommen wurden.

Die in diesem Kapitel enthaltenen Bilder und sonstiges Drittmaterial unterliegen ebenfalls der genannten Creative Commons Lizenz, sofern sich aus der Abbildungslegende nichts anderes ergibt. Sofern das betreffende Material nicht unter der genannten Creative Commons Lizenz steht und die betreffende Handlung nicht nach gesetzlichen Vorschriften erlaubt ist, ist für die oben aufgeführten Weiterverwendungen des Materials die Einwilligung des jeweiligen Rechteinhabers einzuholen.

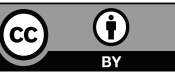

